BULLETIN (New Series) OF THE

AMERICAN MATHEMATICAL SOCIETY

Volume 42, Number 3, Pages 365-393

S 0273-0979(05)01058-X

Article electronically published on April 13, 2005

\title{
CONFORMAL INVARIANTS AND PARTIAL DIFFERENTIAL EQUATIONS
}

\author{
SUN-YUNG ALICE CHANG
}

\section{Contents}

0. Introduction

1. A blow-up sequence of functions, when $n \geq 3$

2. Gaussian curvature equation and the Gauss-Bonnet formula

3. Conformally covariant differential operators and the Q-curvatures

4. Fully nonlinear equations in conformal geometry

5. Boundary operator, Cohn-Vossen inequality

6. Conformal covariant operator and Q-curvature

7. Renormalized volume

References

\section{INTRODUCTION}

Our goal is to study quantities in Riemannian geometry which remain invariant under the "conformal change of metrics"-that is, under changes of metrics which stretch the length of vectors but preserve the angles between any pair of vectors. We call such a quantity "conformally invariant".

In conjunction with the study of conformal invariants, we are also interested in studying "conformally covariant operators", that is, linear differential operators defined on a manifold which prescribes the change of a geometric quantity under conformal change of metrics.

The study of conformal invariants has a long history going back at least to Poincaré and Cartan. In recent years, there has been intensive study of the existence and construction of conformal covariant operators and also applications to problems in geometry and related problems in string theory and mathematical physics. In these talks, I will report some progress in this research area, with emphasis on the PDE approach to these problems.

A model example is that of the Laplace operator $\Delta_{g}$ on a compact surface $M^{2}$ with a Riemannian metric $g$. In this case, under the conformal change of metric $g_{w}=e^{2 w} g, \Delta_{g_{w}}=e^{-2 w} \Delta_{g}$ is a familiar example of a conformally covariant operator.

Received by the editors June 30, 2004.

2000 Mathematics Subject Classification. Primary 53A30, 58J05, 35J60.

The research of Chang is supported in part by NSF Grant DMS-0245266.

This paper is based on a Colloquium Lecture given at the Joint Mathematics Meetings in January 2004 in Phoenix, AZ.

(C)2005 American Mathematical Society Reverts to public domain 28 years from publication 
If we denote $K_{g}$ as the Gaussian curvature of $\left(M^{2}, g\right)$, then according to the wellknown Gauss-Bonnet formula:

$$
2 \pi \chi\left(M^{2}\right)=\int_{M} K_{g} d v_{g}
$$

where $\chi\left(M^{2}\right)$ denotes the Euler characteristic of the surface $M^{2}$. We conclude that the term $\int_{M} K_{g} d v_{g}$ is a topological, hence a conformal, invariant, while $K_{g_{w}}$ and $K_{g}$ are related by the partial differential equation

$$
-\Delta_{g} w+K_{g}=K_{g_{w}} e^{2 w} .
$$

There is extensive literature about the equation (0.1). For example, to solve $K_{g_{w}}=$ $c$ for some constant $c$ is equivalent to the uniformization theorem of surfaces, i.e. the classification of compact surfaces according to the sign of $\mathrm{c}$ to be $+1,0$ or -1 as diffeomorphic to the standard 2-sphere, the 2-torus or a hyperbolic surface. Note in this case, the sign of $c$ is also determined by the sign of the conformal invariant $\int_{M} K_{g} d v_{g}$. It turns out the variational functional to study the equation (0.1) is also the logarithmic determinant of the Laplace operator in the sense of Ray-Singer and coincides with the Polyakov formula for compact surfaces in string theory. We will discuss this in more detail in section 2.

Another well known example is the conformal Laplacian operator $L_{g}=-\Delta_{g}+$ $\frac{n-2}{4(n-1)} R_{g}$ defined on manifolds $\left(M^{n}, g\right)$ of dimension $n \geq 3$, where $R_{g}$ denotes the scalar curvature of the metric $g$. In this case, denoting the conformal change of metric as $g_{u}=u^{\frac{4}{n-2}} g$ for some positive function $u, R_{g_{u}}$ and $R_{g}$ are related by the Yamabe equation:

$$
L_{g} u=\frac{n-2}{4(n-1)} R_{g_{u}} u^{\frac{n+2}{n-2}} .
$$

Recall that the famous Yamabe problem is to solve equation (0.2) for a positive function $u$ with $R_{g_{u}} \equiv c$ where $c$ is a constant. The problem was solved by the series of works of Yamabe, Trudinger, Aubin and Schoen. In particular, in the work of Schoen, he has applied the positive mass theorem to bound the conformal invariantthe Yamabe constant $\Lambda(M, g)$ defined as $\Lambda(M, g)=\inf _{g_{u} \in[g] \text {, vol } g_{u}=1} \int_{M} R_{g_{u}} d V_{g_{u}}$.

Note that on Riemannian manifolds of dimensions $n \geq 3$, there are many other curvature invariants-e.g. sectional curvature, Ricci curvature and Weyl curvature, etc.--which are the various components of the curvature tensor. We remark that the scalar curvature is the trace of the Ricci curvature tensor and the Weyl curvature $W$ satisfies the pointwise conformal invariant property $W_{g_{w}}=e^{-2 w} W_{g}$ under conformal change of metrics $g_{w}=e^{2 w} g$.

It turns out that the Laplacian operator on 2-surfaces and the conformal Laplace operator on manifolds of dimension greater than two are special cases of a general hierarchy of conformally covariant operators. Based on the earlier work (1985) of C. Fefferman and R. Graham on the construction of ambient metric, there has been systematic study of the existence and construction of conformal covariants and conformal invariants of higher orders. In particular, on manifolds of dimension 4, there is a fourth order conformal covariant operator, independently discovered by Paneitz in 1983, with the leading symbol the bi-Laplace operator

$$
P \varphi \equiv \Delta^{2} \varphi+\delta\left(\frac{2}{3} R g-2 \text { Ric }\right) d \varphi
$$


where $\delta$ denotes the divergence, $d$ the deRham differential and Ric the Ricci tensor of the metric. The Paneitz operator on the 4-manifold satisfies the conformal covariant property that $P_{g_{w}}=e^{-4 w} P_{g}$; also the Paneitz operator applied to a conformal factor determines a fourth order curvature invariant which we will call the Q-curvature defined as

$$
Q=-\frac{1}{12}\left(-\Delta P+R^{2}-3|R i c|^{2}\right) .
$$

The relation of $(\mathrm{P}, \mathrm{Q})$ is like that of $(\Delta, \mathrm{K})$ in $(0.2)$ :

$$
P_{g} w+2 Q_{g}=2 Q_{g_{w}} \text {. }
$$

There are two reasons that make this Q-curvature equation attractive to study. The first consideration comes from the analytic point of view, namely that the generic singularities of the Q-curvature equation are isolated points. The second consideration comes from geometry: the Q-curvature prescribed by the Paneitz operator can be viewed as part of the integrand in the Chern-Gauss-Bonnet formula; thus the integration of $Q$ is conformally invariant. Since the Q-curvature contains information about the Ricci tensor, it influences the geometry of the underlying manifold directly.

There is also active development of the study of the structure of the family of conformally covariant operators and their associated conformal invariants by Tom Branson and his collaborators.

The Q-curvature equation is intimately related to a fully non-linear second order elliptic equation. Up to a fourth order divergence term, the Q-curvature is the second elementary symmetric function $\sigma_{2}(A)$ of the Schouten tensor $A_{i j}=R_{i j}-$ $\frac{1}{2(n-1)} R g_{i j}$, where $R_{i j}$ denotes the Ricci tensor and $R$ the scalar curvature of the metric $g$. The positivity of $\sigma_{2}(A)$ implies a sign on the Ricci tensor; hence there is implication for the fundamental group $\pi_{1}(M)$. Thus the $\sigma_{2}(A)$ equation contains geometric information that is absent in the scalar curvature equation. The $\mathrm{Q}$ curvature equation was first used as an approximation to the lower order fully non-linear equation. In the past few years, a number of techniques have been introduced to study the fully non-linear equation associated with prescribing the quantity $\sigma_{k}(A)$.

The Q-curvature equation arises from considerations in conformally compact Einstein manifolds, a subject that was initially developed by C. Fefferman and R. Graham and has now acquired wide interest due to the development of string theory. In particular, it is closely related to the scattering theory of such manifolds.

In the following, I will discuss the recent development around the Q-curvature equation. This will by no means be a general survey of the subject, as I will only report on what I am familiar with.

In the first lecture, I will give a brief survey of the subject. I will then describe the variational approach to studying the equations (0.1) and (0.2). The basic difficulty in finding solutions via this approach is the fact that the conformal group of the standard n-sphere is not compact, thus creating a non-compact family of solutions which does not have a priori $L^{\infty}$ bound and which satisfies $K_{g_{w}} \equiv 1$ for (0.1) or $R_{g_{u}} \equiv n(n-1)$ for $(0.2)$. I will describe the blow-up sequence and the analytic tools (i.e. sharp Sobolev inequality for manifolds of dimension $n \geq 3$ and the Moser-Trudinger inequality on compact surfaces) to overcome the difficulty. To make the lectures expository, in section 1 below I will first describe the behavior 
of the blow-up sequence of functions defined on the Euclidean space $\mathbb{R}^{n}$ for $n \geq 3$, then "transport" the sequence of functions defined on $\mathbb{R}^{n}$ to functions defined on the sphere $S^{n}$ via the stereographic projections. We will then see that the "uniqueness" of this blow-up sequence of functions leads to the solution (through the series of work of Yamabe, Trudinger, T. Aubin and Schoen) of the famous "Yamabe" problem. In section 2, I will explain the corresponding phenomenon on compact surfaces. An added complication in this case is that on domains in $\mathbb{R}^{2}$, the embedding of the Sobolev space $W^{1,2}$ (i.e. functions with first derivatives in $L^{2}$ ) is in the Orlicz space $e^{L^{2}}$, with the best embedding constant studied by Moser. I will briefly describe the Trudinger-Moser inequality and the variational approach to the prescribing Gaussian curvature equations (0.1) and uniformization theorem.

In the second lecture, in sections 3 and 4, I will discuss the study of the Paneitz operator on four manifolds, the Q-curvature, connection of Q-curvature to symmetric functions of the Schouten tensor, and the associated fully non-linear curvature $\sigma_{2}$ equations. I will also discuss some geometric applications. As this is a research field where both the PDE and the geometric aspects of the problems are developing very fast currently, the discussion here will be quite partial. I will also mention some open questions.

Although the existence of $Q$ curvature and its relation to the Gauss-Bonnet integrand are so far restricted to even dimensional manifolds without boundary, there are indications that some generalization of this notion is also interesting for an odd dimensional manifold viewed as the boundary of an even dimensional manifold. We will discuss some aspects of this in section 5 .

In the third lecture, I will report on some recent developments. One particularly interesting one is the work of R. Graham and Zworski relating the existence of the conformal invariants and Q-curvature to the scattering theory of the conformally compact Einstein manifold. I will discuss the concept of the renormalized volume in this setting and some of the many open questions in some recent developments.

These notes are an expanded and modified version of the lecture notes in the Proceedings of ICM 2004, Beijing, written by the author together with Paul Yang. The author is grateful for the input of Paul Yang, together with many co-workers she is fortunate to be associated with. She also would like to acknowledge consultation with Charles Fefferman and Robin Graham for suggestions and comments about the notes.

\section{A BLOW-UP SEQUENCE of FUnCtions, When $n \geq 3$}

In this section we will describe a blow-up sequence of functions that occur for many elliptic equations which enjoy the conformal invariance property in conformal geometry.

We will start by describing the extremals for a Sobolev embedding theorem on the Euclidean space $\mathbb{R}^{n}$ for $n \geq 3$. Recall that for all functions $v \in C_{0}^{\infty}\left(\mathbb{R}^{n}\right)$, the classical Sobolev embedding theorem says that

$$
\Lambda\left(\int_{\mathbb{R}^{n}}|v|^{p} d x\right)^{\frac{2}{p}} \leq \int_{\mathbb{R}^{n}}|\nabla v|^{2} d x .
$$

Or equivalently we say that $W_{0}^{1,2}\left(\mathbb{R}^{n}\right)$ embeds into $L^{p}\left(\mathbb{R}^{n}\right)$. By a dilation of $v(x)$ to $v(\lambda x)$, we see that the optimal exponent $p$ in $(1.1)$ is $p=\frac{2 n}{n-2}$. 
It turns out that we can find the best constant $\Lambda$ and the extremal functions $v$ which satisfy the inequality in (1.1). To do so, we suppose $v(x)=v(|x|)=v(r)$ is a radially symmetric extremal function; then it satisfies the ordinary differential equation

One solution is

$$
\left\{\begin{array}{l}
v^{\prime \prime}+\frac{n-1}{r} v^{\prime}+m v^{\frac{n+2}{n-2}}=0 \\
v(0)=a, v^{\prime}(0)=0
\end{array}\right.
$$

$$
\left\{\begin{array}{l}
\Lambda(x)=\left(\frac{2}{1+|x|^{2}}\right)^{\frac{n-2}{2}} \\
a=2^{\frac{n-2}{2}}, m=\frac{n(n-2)}{4} .
\end{array}\right.
$$

We then observe that the inequality is invariant under the following translation and dilations of the function $v$ :

$$
v \rightarrow v_{\epsilon}(x)=\epsilon^{\frac{2-n}{2}} v\left(\frac{x-x_{0}}{\epsilon}\right),
$$

where $\epsilon>0$ and $x_{0}$ is any point in $\mathbb{R}^{n}$. In other words, we have

$$
v_{\epsilon}(x)=\left(\frac{2 \epsilon}{\epsilon^{2}+\left|x-x_{0}\right|^{2}}\right)^{\frac{n-2}{2}}
$$

are all extremals for the Sobolev embedding (1.1), and we have the following remarkable theorem. In stating the theorem, we have assumed that we fixed the class of functions $v \in W_{0}^{1,2}\left(\mathbb{R}^{n}\right)$ with $L^{\frac{2 n}{n-2}}$ norm as that of the function $v_{1}$.

Theorem 1.1 (9], 97], 4]). The best constant in the Sobolev inequality in (1.1) is $\Lambda=\frac{n(n-2)}{4} \omega_{n}^{2 / n}$ where $\omega_{n}$ is the surface area of the unit sphere $S^{n}$. It is (only) realized by the functions $v_{\epsilon}$ as described in the above.

We now fix $x_{0}=0$ and observe that the sequence of functions $v_{\epsilon}$ has the following properties:

(i) $v_{\epsilon}(0)=\left(\frac{2}{\epsilon}\right)^{\frac{n-2}{2}} \rightarrow \infty$ as $\epsilon \rightarrow 0$;

(ii) $v_{\epsilon}(x) \rightarrow 0$, for all $x \neq 0$, as $\epsilon \rightarrow 0$;

(iii) $\int_{\mathbb{R}^{n}}\left|v_{\epsilon}(x)\right|^{\frac{2 n}{n-2}} d x=\int_{\mathbb{R}^{n}}\left|v_{1}(x)\right|^{\frac{2 n}{n-2}} d x$, for all $\epsilon>0$;

(iv) $\int_{\mathbb{R}^{n}}\left|\nabla v_{\epsilon}(x)\right|^{2} d x=\int_{\mathbb{R}^{n}}\left|\nabla v_{1}(x)\right|^{2} d x$, for all $\epsilon>0$.

Thus $v_{\epsilon}$ is a sequence of functions which is bounded in $W^{1,2}\left(\mathbb{R}^{n}\right)$, and the weak limit of the sequence is the zero function; hence it does not have a convergent subsequence in $L^{\frac{2 n}{n-2}}$. In other words, we are saying that the embedding of the Sobolev space $W^{1,2}\left(\mathbb{R}^{n}\right)$ into $L^{\frac{2 n}{n-2}}$ is not compact. This lack of compactness turns out to be at the heart of the problem.

The Euler Lagrange equation for the extremal function saturating the inequality (1.1) is

$$
-\Delta v=\frac{n(n-2)}{4} v^{\frac{n+2}{n-2}} \text { on } \mathbb{R}^{n} .
$$

Thus functions $v_{\epsilon}$ above are solutions of the equation (1.2). According to a result of Caffarelli-Gidas-Spruck ([18), any positive solution of (1.2) is one of the $v_{\epsilon}$ as above. In other words, the solutions of (1.2) are unique up to dilations and translations.

The same blow-up sequence may be realized on the unit sphere $S^{n}$ using the stereographic projection. To see this, for each point $\xi \in S^{n}$, denote its corresponding point under the stereographic projection $\pi$ from $S^{n}$ to $\mathbb{R}^{n}$, sending the north pole on $S^{n}$ to $\infty$. That is: suppose $\xi=\left(\xi_{1}, \xi_{2}, \ldots, \xi_{n+1}\right)$ is a point in $S^{n} \subset \mathbb{R}^{n+1}$, $x=\left(x_{1}, x_{2}, \ldots, x_{n}\right)$; then $\xi_{i}=\frac{2 x_{i}}{1+|x|^{2}}$ for $1 \leq i \leq n, \xi_{n+1}=\frac{|x|^{2}-1}{|x|^{2}+1}$. Suppose $u$ is a 
smooth function defined on $S^{n}$, noting that the Jacobian of $\pi^{-1}$ is $J_{\pi^{-1}}=\frac{2}{1+|x|^{2}}$; thus if we denote

$$
v(x)=u(\xi(x))\left(\frac{2}{1+|x|^{2}}\right)^{\frac{n-2}{2}},
$$

then inequality (1.1) is equivalent to

$$
\Lambda\left(\int_{S^{n}}|u(\xi)|^{\frac{2 n}{n-2}} d v(\xi)\right)^{\frac{n-2}{2}} \leq \int_{S^{n}}|\nabla u(\xi)|^{2} d v(\xi)+\frac{n(n-2)}{4} \int_{S^{n}}|u(\xi)|^{2} d v(\xi),
$$

where $d v(\xi)=\left(\frac{2}{1+|x|^{2}}\right)^{n}$ is the standard volume form on the unit sphere $S^{n}$.

The transformed function $u(\xi)$ satisfies the equation:

$$
-\Delta_{g} u+\frac{n(n-2)}{4} u=\frac{n(n-2)}{4} u^{\frac{n+2}{n-2}} \text { on } S^{n},
$$

where $\Delta_{g}$ denotes the Laplace Beltrami operator with respect to the standard metric $g$ on $S^{n}$.

On manifolds $\left(M^{n}, g\right)$ of dimensions greater than two, the conformal Laplacian $L_{g}$ is defined as $L_{g}=-\Delta_{g}+c_{n} R_{g}$ where $c_{n}=\frac{n-2}{4(n-1)}$ and $R_{g}$ denotes the scalar curvature of the metric $g$. An analogue of equation (1.2) is the equation, commonly referred to as the Yamabe equation, which relates the scalar curvature under the conformal change of metric to the background metric. In this case, it is convenient to denote the conformal metric as $\bar{g}=u^{\frac{4}{n-2}} g$ for some positive function $u$; then the equation becomes

$$
L_{g} u=c_{n} \bar{R} u^{\frac{n+2}{n-2}} .
$$

Thus a positive solution $u$ on the sphere of equation (1.4) gives a metric $u^{\frac{4}{n-2}} g$ of constant scalar curvature $n(n-1)$. In fact the functions $u_{\epsilon}$ obtained from $v_{\epsilon}$ are the only positive solutions of (1.4). This follows from the result of Caffarelli-GidasSpruck which we have just discussed.

The famous Yamabe problem to solve (1.5) with $\bar{R}$ a constant has been settled by Yamabe ([103]), Trudinger ([99]), Aubin ([5]) and Schoen ([93]). To solve the equation (1.5) by the variational method, one has to prove the minimal solution is attained by the extremal function $u$ extremizing the inequality

$$
\Lambda_{g}\left(\int_{M}|u|^{\frac{2 n}{n-2}} \mid d v_{g}\right)^{\frac{n-2}{2}} \leq \int_{M}\left|\nabla_{g} u\right|^{2} d v_{g}+c_{n} \int_{M} R|u|^{2} d v_{g},
$$

for some constant $\Lambda_{g} \leq \Lambda$. This constant $\Lambda_{g}$ is called the Yamabe constant and is an invariant of the conformal structure. A crucial ingredient in the solution is to establish criteria for compactness of the minimizing sequence; such criteria involve conformal invariants which serve to distinguish the conformal structure from that of the standard sphere. This is a common strategy in treating all the conformally covariant differential equations that we will encounter. In the solution by Aubin ([5]), the non-vanishing of the Weyl tensor (a local conformal invariant) in high dimensions plays this role. The remaining case requires a global invariant. In a remarkable paper, Schoen ([93]) uses the positive mass theorem to differentiate the conformal structure from the standard n-sphere.

In the past two decades, there has been extensive study of the Yamabe equation and more generally the equation to prescribe the scalar curvature function by many different groups of mathematicians. We will not be able to survey all the results here. We will just mention that for the degree theory for the existence of solutions 
for a given function $R$ on the n-sphere, there is work of Bahri-Coron ([7]), ChangGursky-Yang (24]) and Schoen-Zhang (94]) for $n=3$ and under further constraints on the functions for $n \geq 4$ by Y. Li ([74]) and by C.-C. Chen and C.-S. Lin ([38]).

\section{Gaussian curvature equation and the Gauss-Bonnet Formula}

We now discuss the situation in dimension two. On a bounded domain $D$ in $\mathbb{R}^{2}$, a function in $W_{0}^{1,2}(D)$ is in $L^{p}(D)$ for all $p>0$, yet it is easy to see that such a function may not be bounded. For example, take $D$ to be the unit ball $B$ in $R^{2}$; then $w(x)=\log \log \left(e-1+\frac{1}{|x|}\right)$ is such a function.

Theorem 2.1 ([79, 98]). Suppose $D$ is a smooth domain in $\mathbb{R}^{2}$. Then there is a constant $C$ so that for all functions $w \in W_{0}^{1,2}(D)$ with $\int_{D}|\nabla w(x)|^{2} d x \leq 1$, we have

$$
\int_{D} e^{\alpha w^{2}}(x) d x \leq C|D|
$$

for any $\alpha \leq 4 \pi$, with $4 \pi$ being the best constant. I.e. if $\alpha>4 \pi$, the integral can be made arbitrarily large by appropriate choice of $w$, where $|D|$ denotes the Lebesgue measure of $D$.

Remark. We have quoted here a special case of the original theorem. Inequality (2.1) above is the limiting case of the Sobolev embedding: $W_{0}^{1, q}(D) \hookrightarrow L^{p}$ with $\frac{1}{p}=\frac{1}{q}-\frac{1}{n}$ for $q<n$ and for bounded domain $D$ in $\mathbb{R}^{n}$. Moser has also established a similar inequality for all $n \geq 2$. The result was also generalized to the limiting case of $W^{\alpha, p}$ embeddings with $\alpha p=n$ by Adams ([1]). Subsequently, Carleson and Chang ([20]) found that, contrary to the situation for the Sobolev embedding, there is an extremal function realizing the maximum value of the inequality of Moser when the domain is the unit ball in Euclidean space. This fact remains true for simply connected domains in the plane (Flücher [48]) and for some domains in the n-sphere (Soong [66).

The following linearized form of the inequality (2.1) is particularly useful.

\section{Corollary 1.}

$$
\log \frac{1}{|D|} \int_{D} e^{2 w} d x \leq \frac{1}{4 \pi} \int_{D}|\nabla w|^{2} d x .
$$

Extremal functions for both inequalities (2.1) and (2.2) are interesting to study (see [20], 22]). Another result is the following uniqueness theorem of W.X. Chen and $\mathrm{C}$. $\mathrm{Li}$, the proof of which was based on a beautiful application of the method of moving planes.

Theorem $2.2\left([36)\right.$. Suppose $w$ is in $C^{2}\left(\mathbb{R}^{2}\right)$, with $e^{2 w} \in L^{1}\left(\mathbb{R}^{2}\right)$, and satisfies the equation

$$
-\Delta w=e^{2 w} \text { on } \mathbb{R}^{2} .
$$

Then

$$
w(x)=\log \frac{2 \epsilon}{\epsilon^{2}+\left|x-x_{0}\right|^{2}}
$$

for some $\epsilon>0$ and some $x_{0} \in \mathbb{R}^{2}$. 
As we will see below after pulling functions from the 2-sphere $S^{2}$ to $\mathbb{R}^{2}$, the corresponding theorem is a statement of the "uniqueness" of metrics conformal to the standard sphere $\left(S^{2}, g\right)$ with Gaussian curvature identically equal to one-those are metrics which are pullbacks $T^{*}(g)$ of the standard metric for some Mobius transformation $T$ of $S^{2}$.

We now describe the situation on compact surfaces. On a compact surface $(M, g)$ with a Riemannian metric $g$, a natural curvature invariant associated with the Laplace operator $\Delta=\Delta_{g}$ is the Gaussian curvature $K=K_{g}$. Under the conformal change of metric $g_{w}=e^{2 w} g$, we have

$$
-\Delta_{g} w+K_{g}=K_{w} e^{2 w} \text { on } M
$$

where $K_{w}$ denotes the Gaussian curvature of $\left(M, g_{w}\right)$. The classical uniformization theorem to classify compact closed surfaces can be viewed as finding a solution of equation (2.4) with $K_{w} \equiv-1,0$, or 1 according to the sign of $\int K d v_{g}$. Recall that the Gauss-Bonnet theorem states

$$
2 \pi \chi(M)=\int_{M} K_{w} d v_{g_{w}}
$$

where $\chi(M)$ is the Euler characteristic of $M$, a topological invariant. The variational functional with (2.4) as Euler equation for $K_{w}=$ constant is thus given by

$$
J[w]=\int_{M}|\nabla w|^{2} d v_{g}+2 \int_{M} K w d v_{g}-\left(\int_{M} K d v_{g}\right) \log \frac{\int_{M} d v_{g_{w}}}{\int_{M} d v_{g}} .
$$

When the surface $(M, g)$ is the standard 2-sphere $S^{2}$ with the standard canonical metric, the problem of prescribing Gaussian curvature on $S^{2}$ is commonly known as the Nirenberg problem. For general simply connected compact surface $M$, Kazdan and Warner ([71]) gave a necessary and sufficient condition for the function when $\chi(M)=0$ and some necessary condition for the function when $\chi(M)<0$. They also pointed out that in the case when $\chi(M)>0$, i.e. when $(M, g)=\left(S^{2}, g_{c}\right)$, the standard 2-sphere with the canonical metric $g=g_{c}$, there is an obstruction for the problem:

$$
\int_{S^{2}} \nabla K_{w} \cdot \nabla x e^{2 w} d v_{g}=0
$$

where $x$ is any of the ambient coordinate functions. Moser ([80]) realized that this implicit integrability condition is satisfied if the conformal factor has antipodal symmetry. He proved for an even function $f$ that the only condition for (2.4) to be solvable with $K_{w}=f$ is the necessary condition that $f$ be positive somewhere. An important tool introduced by Moser is the following inequality ([79]), which is the analogue of inequality (2.1) on the 2 -sphere.

Let $w$ be a smooth function on the 2-sphere satisfying the normalized conditions:

$$
\int_{S^{2}}|\nabla w|^{2} d v_{g} \leq 1 \text { and } \bar{w}=0
$$

where $\bar{w}$ denotes the mean value of $w$; then

$$
\int_{S^{2}} e^{\beta w^{2}} d v_{g} \leq C
$$

where $\beta \leq 4 \pi$ and $C$ is a fixed constant and $4 \pi$ is the best constant. If $w$ has antipodal symmetry, then the inequality holds for $\beta \leq 8 \pi$. Actually the best 
constant $\beta$ is the isoperimetric constant for the class of functions (cf. 34]). Based on the inequality of Moser and subsequent work of Aubin ([5]) and Onofri ([82]), we devised a degree count ([33], [34], [24]) associated to the function $f$ and the Mobius group on the 2-sphere that is motivated by the Kazdan-Warner condition (2.7). This degree actually computes the Leray-Schauder degree of the equation (2.4) as a non-linear Fredholm equation. In the special case that $f$ is a Morse function satisfying the condition $\Delta f(x) \neq 0$ at the critical points $x$ of $f$, this degree can be expressed as:

$$
\sum_{\nabla f(q)=0, \Delta f(q)<0}(-1)^{i n d(q)}-1 .
$$

The latter degree count is also obtained later by K.-C. Chang and Liu ([21]) and Han $([65])$.

There is a beautiful extension of this existence theory to the more general setting of the so-called mean field equation by the work of C.-C. Chen and C.-S. Lin (37) in which a degree count is derived for the following equation on any compact Riemann surface:

$$
\Delta u+\rho\left(\frac{h e^{u}}{\int_{M} h e^{u}}\right)-1=0
$$

where $h$ is a positive function and $\rho$ is any given positive parameter.

There is an important geometric meaning of the functional $J$ given by Ray-Singer (92]) and Polyakov ([90]) (see also Okikiolu [85]):

$$
J[w]=12 \pi \log \left(\frac{\operatorname{det} \Delta_{g}}{\operatorname{det} \Delta_{g_{w}}}\right)
$$

for metrics $g_{w}$ where the volume of $g_{w}$ equals the volume of $g$ and where the determinant of the Laplacian det $\Delta_{g}$ is defined by Ray-Singer via the "regularized" zeta function. In ([82]) (see also Hong [69]), Onofri established the sharp inequality that on the 2-sphere

$$
J[w] \geq 0
$$

and $J[w]=0$ precisely for conformal factors $w$ of the form $e^{2 w} g=T^{*} g$ where $T$ is a Mobius transformation of the 2-sphere. Later Osgood-Phillips-Sarnak ([83], 84]) arrived at the same sharp inequality in their study of heights of the Laplacian. This inequality also plays an important role in their proof of the $C^{\infty}$ compactness of isospectral metrics on compact surfaces.

The formula of Polyakov-Ray-Singer has been generalized to manifolds of dimension greater than two in many different settings, one of which we will discuss in section 2 below. There is also a general study of extremal metrics for det $\Delta_{g}$ or det $L_{g}$ for metrics $g$ in the same conformal class with a fixed volume or for all metrics with a fixed volume ([8], [13], 12], [86]). In terms of comparing determinants of the Laplacian of metrics across different conformal classes, there is the remarkable result of Okikiolu ([86]) that among all metrics with the same volume as the standard metric on the 3 -sphere, the standard canonical metric is a local maximum for the functional det $\Delta_{g}$. 


\section{Conformally COVARIANT DifFERENTIAL OPERATORS AND THE Q-CURVATURES}

We have seen that in dimension two, under the conformal change of metrics $g_{w}=e^{2 w} g$, the associated Laplacians are related by

$$
\Delta_{g_{w}}=e^{-2 w} \Delta_{g}
$$

Similarly on $\left(M^{n}, g\right)$, the conformal Laplacian $L=-\Delta+c_{n} R$, where $c_{n}=\frac{n-2}{4(n-1)}$, transforms under the conformal change of metric $\bar{g}=u^{\frac{4}{n-2}} g$ :

$$
L_{\bar{g}}=u^{-\frac{n+2}{n-2}} L_{g}(u \cdot) .
$$

In general, we call a metrically defined operator $A$ conformally covariant of bi-degree $(a, b)$ if under the conformal change of metric $g_{\omega}=e^{2 \omega} g$, the pair of corresponding operators $A_{g_{\omega}}$ and $A$ are related by

$$
A_{g_{\omega}}(\varphi)=e^{-b \omega} A\left(e^{a \omega} \varphi\right) \text { for all } \varphi \in C^{\infty}\left(M^{n}\right) .
$$

Thus the conformal Laplacian operator is conformally covariant of bi-degree $\left(\frac{n-2}{2}\right.$, $\left.\frac{n+2}{2}\right)$.

There are many operators besides the Laplacian $\Delta$ on compact surfaces and the conformal Laplacian $L$ on general compact manifold of dimension greater than two which have the conformal covariance property. We begin with the fourth order operator on 4-manifolds discovered by Paneitz ([89]) in 1983:

$$
P \varphi \equiv \Delta^{2} \varphi+\delta\left(\frac{2}{3} R g-2 \mathrm{Ric}\right) d \varphi
$$

where $\delta$ denotes the divergence, $d$ the deRham differential and Ric the Ricci tensor of the metric. The Paneitz operator $P$ (which we will later denote by $P_{4}$ ) is conformally covariant of bi-degree $(0,4)$ on 4 -manifolds; i.e.

$$
P_{g_{w}}(\varphi)=e^{-4 \omega} P_{g}(\varphi) \text { for all } \varphi \in C^{\infty}\left(M^{4}\right) \text {. }
$$

The Paneitz operator acting on functions defined on 4-manifolds behaves much like the Laplace operator acting on functions defined on surfaces. For example, there is a $Q$-curvature defined as

$$
Q=\frac{1}{12}\left(-\Delta R+R^{2}-3|R i c|^{2}\right) .
$$

The relation of the pair $(P, Q)$ is like that of $(-\Delta, K)$ in $(2.4)$ :

$$
P_{g} w+2 Q_{g}=2 Q_{g_{w}} e^{4 w} .
$$

In addition, $Q$ can be viewed as part of the 4-dimensional Chern-Gauss-Bonnet formula:

$$
8 \pi^{2} \chi\left(M^{4}\right)=\int\left(\frac{1}{4}|W|^{2}+2 Q\right) d v
$$

where $W$ denotes the Weyl tensor. Note that $|W|^{2} d v$ is a pointwise conformal invariant; thus the curvature integral $\int Q d v$ is a conformal invariant.

A basic existence result for the $Q$-curvature equation, based on a result of Gursky (60]), is given by the following theorem. 
Theorem 3.1. On $\left(M^{4}, g\right)$

(i) 60

If the Yamabe constant $\Lambda(M, g) \geq 0$, and $\int_{M} Q_{g} d v_{g} \geq 0$, then $P$ is a positive operator with its kernel consisting of constants.

(ii) 60.

Under the condition of (i), $\int_{M} Q_{g} d v_{g} \leq 8 \pi^{2}$, with equality if and only if $(M, g)$ is conformally equivalent to the standard 4-sphere.

(iii) 35

If $\int_{M} Q_{g} d v_{g}<8 \pi^{2}$ and the $P$ operator is positive except for constants, then equation (3.5) may be solved with $Q_{g_{w}}$ given by a constant.

We remark that the conditions given by Gursky in (i) above are remarkable, as both $\Lambda(M, g)$ and $\int_{M} Q_{g} d v_{g}$ are conformally invariant quantities; thus both are natural conditions for the positivity of the conformally covariant Paneitz operator. The condition (ii) in the theorem above can be viewed as the analogue of the positive mass theorem that is the source for the basic compactness result for the $Q$-curvature equation as well as the associated fully non-linear second order equations that we discuss in section 4. Gursky's argument is based on a more general existence result in which we consider a family of fourth order equations

$$
\gamma_{1}|W|^{2}+\gamma_{2} Q-\gamma_{3} \Delta R=\frac{\bar{k}}{\mathrm{Vol}}
$$

where $\bar{k}=\int\left(\gamma_{1}|W|^{2}+\gamma_{2} Q\right) d v$. These equations typically arise as the Euler equations of the functional determinants. For a conformally covariant operator $A$ of bi-degree $(a, b)$ with $b-a=2$ (e.g. when $A=L$ is the conformal Laplace operator) Branson and Ørsted ([14]) gave an explicit computation of the normalized form of $\log \frac{\operatorname{det} A_{w}}{\operatorname{det} A}$ which may be expressed as:

$$
F[w]=\gamma_{1} I[w]+\gamma_{2} I I[w]+\gamma_{3} I I I[w]
$$

where $\gamma_{1}, \gamma_{2}, \gamma_{3}$ are constants depending only on $A$ and

$$
\begin{aligned}
I[w] & =4 \int|W|^{2} w d v-\left(\int|W|^{2} d v\right) \log \frac{\int e^{4 w} d v}{\int d v}, \\
I I[w] & =\langle P w, w\rangle+4 \int Q w d v-\left(\int Q d v\right) \log \frac{\int e^{4 w} d v}{\int d v}, \\
I I I[w] & =\frac{1}{3}\left(\int R_{g_{w}}^{2} d v_{g_{w}}-\int R^{2} d v\right) .
\end{aligned}
$$

In 35, we gave the general existence result:

Theorem 3.2. If the functional $F$ satisfies $\gamma_{2}>0, \gamma_{3}>0$, and $\bar{k}<8 \gamma_{2} \pi^{2}$, then $\inf _{w \in W^{2,2}} F[w]$ is attained by some function $w_{d}$ and the metric $g_{d}=e^{2 w_{d}} g_{0}$ satisfies the equation

$$
\gamma_{1}|W|^{2}+\gamma_{2} Q_{d}-\gamma_{3} \triangle_{d} R_{d}=\frac{\bar{k}}{\operatorname{Vol}\left(g_{d}\right)}
$$

Furthermore, $g_{d}$ is smooth.

This existence result is based on extensions of Moser's inequality by Adams ([1]) to operators of higher order. In the special case of $\left(M^{4}, g\right)$, the inequality states 
that for functions in the Sobolev space $W^{2,2}(M)$ with $\int_{M}(\Delta w)^{2} d v_{g} \leq 1$, and $\bar{w}=0$, we have

$$
\int_{M} e^{32 \pi^{2} w^{2}} d v_{g} \leq C,
$$

for some constant $C$. There are several applications of these existence results to the study of conformal structures in dimension $n=4$. In section 4 below we will discuss the use of such fourth order equations as regularization of the more natural fully non-linear equations concerned with the Schouten tensor. Here we will mention some elegant applications by M. Gursky ([59]) to characterize a number of extremal conformal structures.

Theorem 3.3. Suppose $(M, g)$ is a closed oriented manifold of dimension four with positive Yamabe constant.

(i) If $\int Q_{g} d v_{g}=0$, then $M$ admits a non-zero harmonic 1-form if and only if $(M, g)$ is conformal equivalent to a quotient of the product space $S^{3} \times \mathbb{R}$. In particular $(M, g)$ is locally conformally flat.

(ii) If $b_{2}^{+}>0$ (i.e. the intersection form has a positive element), then with respect to the decomposition of the Weyl tensor into the self-dual and anti-self-dual components $W=W^{+} \oplus W^{-}$,

$$
\int_{M}\left|W_{g}^{+}\right|^{2} d v_{g} \geq \frac{4 \pi^{2}}{3}(2 \chi+3 \tau),
$$

where $\tau$ is the signature of $M$. Moreover the equality holds if and only if $g$ is conformal to a (positive) Kahler-Einstein metric.

In the same article ([89]), Paneitz has actually introduced for each manifold of dimension $n, n \neq 2$, some fourth order operators with some conformal covariant property. The relation of these operators to some curvature functions (which we will call the $Q$-curvatures) was later introduced by T. Branson ([10, [1] $)$. When $n \neq 2$, we will call this fourth order Paneitz operator the conformal Paneitz operator:

$$
P_{4}^{n}=\Delta^{2}+\delta\left(a_{n} R g+b_{n} \operatorname{Ric}\right) d+\frac{n-4}{2} Q_{4}^{n}
$$

where

$$
Q_{4}^{n}=c_{n}|R i c|^{2}+d_{n} R^{2}-\frac{1}{2(n-1)} \Delta R
$$

where $a_{n}, b_{n}, c_{n}$ and $d_{n}$ are dimension constants. The conformal Paneitz operator is conformally covariant of bi-degree $\left(\frac{n-4}{2}, \frac{n+4}{2}\right)$.

In dimensions higher than four, the conformal Paneitz operator is being investigated by a number of authors. In particular, Djadli-Hebey-Ledoux ([40]) studied the question of coercivity of the operators $P$ as well as the positivity of the solution functions; Djadli-Malchiodi-Ahmedou ([4]) and Hebey-Robert ([67]) have studied the blow-up analysis of the Paneitz equation. A serious difficulty in dealing with higher order operators is the lack of a good maximum principle. For example it is not clear at all that the minimizer of the corresponding Sobolev quotient is necessarily positive. It is curious to note in this connection that this problem does not arise in dimensions three and four due to the special form of the non-linearity. In dimension three, the fourth order Paneitz equation involves a negative exponent and has many properties which are distinct from their counterparts in higher 
dimensions. The corresponding Sobolev inequality has the form

$$
\Lambda \leq\left(\int P u \cdot u d v\right)\left(\int u^{-6} d v\right)^{-1 / 3}
$$

for all positive smooth functions $u$.

Due to the negative power non-linearity, it is not possible to reduce such an inequality to a domain in Euclidean 3-space. There is partial progress in understanding this equation in the case when the fourth order Paneitz operator is positive ([102]) and in the case where the Paneitz operator satisfies a weak form of positivity (66]).

In general dimensions, there is a hierarchy of higher order operators enjoying conformal covariance. In particular in even dimensions, there are n-th order Paneitz operators and associated $Q$-curvature equations. It is interesting that although the operator is not known explicitly yet (see section 6), there is a general existence result extending Theorem (3.1) which was obtained recently by S. Brendle (15) using a heat flow associated to the $Q$-curvature.

In section 6 , we will discuss the existence theory of local conformal invariants, covariant operators and $Q$-curvatures in general manifolds. The existence results are based on the work of Fefferman and Graham ( $[45)$ and also the recent work of Graham and Zworski ([54).

\section{Fully nONLINEAR EQUATIONS in CONFORMAL GEOMETRY}

In dimensions greater than two, the natural curvature invariants in conformal geometry are the Weyl tensor $W$ and the Schouten tensor $A=R i c-\frac{R}{2(n-1)} g$ that occur in the decomposition of the curvature tensor, where Ric denotes the Ricci curvature tensor:

$$
R m=W \oplus \frac{1}{n-2} A \oplus g
$$

Since the Weyl tensor $W$ transforms by scaling under conformal change $g_{w}=e^{2 w} g$, only the Schouten tensor depends on the derivatives of the conformal factor. It is thus natural to consider $\sigma_{k}\left(A_{g}\right)$ the k-th symmetric function of the eigenvalues of the Schouten tensor $A_{g}$ as curvature invariants of the conformal metrics. As a differential invariant of the conformal factor $w, \sigma_{k}\left(A_{g_{w}}\right)$ is a fully non-linear expression involving the Hessian and the gradient of the conformal factor $w$. We have abbreviated $A_{w}$ for $A_{g_{w}}$ :

$$
A_{w}=(n-2)\left\{-\nabla^{2} w+d w \otimes d w-\frac{|\nabla w|^{2}}{2}\right\}+A_{g} .
$$

The equation

$$
\sigma_{k}\left(A_{w}\right)=1
$$

is a fully non-linear version of the Yamabe equation. For example, when $k=$ $1, \sigma_{1}\left(A_{g}\right)=\frac{n-2}{2(n-1)} R_{g}$, where $R_{g}$ is the scalar curvature of $(M, g)$ and equation (4.3) is the Yamabe equation which we have discussed in section 1. When $k=2$, $\sigma_{2}\left(A_{g}\right)=\frac{1}{2}\left(\mid\right.$ Trace $\left.\left.A_{g}\right|^{2}-\left|A_{g}\right|^{2}\right)=\frac{n}{8(n-1)} R^{2}-\frac{1}{2} \mid$ Ric $\left.\right|^{2}$. In the case when $k=n$, $\sigma_{n}\left(A_{g}\right)=$ determinant of $A_{g}$, an equation of Monge-Ampère type. To illustrate 
that (4.3) is a fully non-linear elliptic equation, we have for example when $n=4$,

$$
\begin{aligned}
\sigma_{2}\left(A_{w}\right) e^{4 w} & =\sigma_{2}\left(A_{g}\right)+2\left((\Delta w)^{2}-\left|\nabla^{2} w\right|^{2}\right. \\
& \left.+\left(\nabla w, \nabla|\nabla w|^{2}\right)+\Delta w|\nabla w|^{2}\right) \\
& + \text { lower order terms }
\end{aligned}
$$

where all derivatives are taken with respect to the metric $g$.

For a symmetric $n \times n$ matrix $M$, we say $M \in \Gamma_{k}^{+}$in the sense of Gårding ([49]) if $\sigma_{k}(M)>0$ and $M$ may be joined to the identity matrix by a path consisting entirely of matrices $M_{t}$ such that $\sigma_{k}\left(M_{t}\right)>0$. There is a rich literature concerning the equation

$$
\sigma_{k}\left(\nabla^{2} u\right)=f,
$$

for a positive function $f$. In the case when $M=\left(\nabla^{2} u\right)$ for convex functions $u$ defined on the Euclidean domains, regularity theory for equations of $\sigma_{k}(M)$ has been well established for $M \in \Gamma_{k}^{+}$for Dirichlet boundary value problems by CaffarelliNirenberg-Spruck ([17]); for a more general class of fully non-linear elliptic equations not necessarily of divergence form by Krylov ([72]), Evans ([43]); and for MongeAmpère equations by Pogorelov ([88) and Caffarelli ([16]). The Monge-Ampère equation for prescribing the Gauss-Kronecker curvature for convex hypersurfaces has been studied by Guan-Spruck ([55]). Some of the techniques in this work can be modified to study equation (4.3) on manifolds. However there are features of the equation (4.3) that are distinct from the equation (4.5). For example, the conformal invariance of the equation (4.3) introduces a non-compactness due to the action of the conformal group that is absent for the equation (4.5).

When $k \neq \frac{n}{2}$ and the manifold $(M, g)$ is locally conformally flat, the equation (4.3) is the Euler equation of the variational functional $\int \sigma_{k}\left(A_{w}\right) d v_{g_{w}}([100])$. In the exceptional case $k=n / 2$, the integral $\int \sigma_{k}\left(A_{g}\right) d v_{g}$ is a conformal invariant. We say $g \in \Gamma_{k}^{+}$if the corresponding Weyl-Schouten tensor $A_{g}(x) \in \Gamma_{k}^{+}$for every point $x \in M$. For $k=1$ the Yamabe equation (1.5) for prescribing scalar curvature is a semi-linear one; hence the condition for $g \in \Gamma_{1}^{+}$is the same as requiring the operator $L_{g}=-\frac{4(n-1)}{n-2} \Delta_{g}+R_{g}$ be a positive operator. The existence of a metric with $g \in \Gamma_{k}^{+}$implies a sign for the curvature functions ([61], [26], [56]).

In dimension 3 , one can characterize metrics with constant sectional curvatures (i.e. space forms) through the study of $\sigma_{2}$.

Theorem 4.1 (61]). On a compact 3-manifold, for any Riemannian metric $g$, denote $\mathcal{F}_{2}[g]=\int_{M} \sigma_{2}\left(A_{g}\right) d v_{g}$. Then a metric $g$ with $\mathcal{F}_{2}[g] \geq 0$ is critical for the functional $\mathcal{F}_{2}$ restricted to the class of metrics with volume one if and only if $g$ has constant sectional curvature.

The criterion for existence of a conformal metric $g \in \Gamma_{k}^{+}$is not easy for $k>1$ since the equation is a fully non-linear one. However when $n=4, k=2$ the invariance of the integral $\int \sigma_{2}\left(A_{g}\right) d v_{g}$ is a reflection of the Chern-Gauss-Bonnet formula

$$
8 \pi^{2} \chi(M)=\int_{M}\left(\frac{1}{4}\left|W_{g}\right|^{2}+\sigma_{2}\left(A_{g}\right)\right) d v_{g} .
$$

In this case it is possible to find a criterion: 
Theorem 4.2 ([26], 62]). For a closed 4-manifold $(M, g)$ satisfying the following conformally invariant conditions:

(i) $\Lambda(M, g)>0$, and

(ii) $\int \sigma_{2}\left(A_{g}\right) d v_{g}>0$,

there exists a conformal metric $g_{w} \in \Gamma_{2}^{+}$.

Remark. In dimension four, the condition $g \in \Gamma_{2}^{+}$implies that $R>0$ and Ricci curvature is positive everywhere. Thus such manifolds have finite fundamental groups. In addition, the Chern-Gauss-Bonnet formula and the signature formula show that this class of 4-manifolds satisfies the same conditions as that of Einstein manifolds with positive scalar curvatures. Thus it is the natural class of 4-manifolds in which to seek an Einstein metric.

In the proof in (26]), the existence result depends strongly on the positivity of the Paneitz operator. The method of continuity is used to consider the deformations of the equation:

$$
(*)_{\delta}: \quad \sigma_{2}\left(A_{g}\right)=\frac{\delta}{4} \Delta_{g} R_{g}-2 \gamma\left|W_{g}\right|^{2}
$$

where $\gamma$ is chosen so that $\int \sigma_{2}\left(A_{g}\right) d v_{g}=-2 \gamma \int\left|W_{g}\right|^{2} d v_{g}$, for $\delta \in(0,1]$ and $\delta$ tends to zero.

In the recent paper (62]), there is an alternative deformation argument giving a more direct proof of the result in Theorem 4.3. The proof relies only on estimates of second order fully non-linear elliptic PDE developed in the recent work of ([76, [57]).

The equation (4.3) becomes meaningful for 4-manifolds which admit a metric $g \in \Gamma_{2}^{+}$. In the article (27]), when the manifold $(M, g)$ is not conformally equivalent to $\left(S^{4}, g_{c}\right)$, we provide a priori estimates for solutions of the equation $\sigma_{2}\left(A_{g_{w}}\right)=f$, where $f$ is a given positive smooth function. Then we apply the degree theory for fully non-linear elliptic equations to the following 1-parameter family of equations:

$$
\sigma_{2}\left(A_{g_{t}}\right)=t f+(1-t)
$$

to deform the original metric to one with constant $\sigma_{2}\left(A_{g}\right)$.

In terms of geometric application, this circle of ideas may be applied to characterize a number of interesting conformal classes in terms of the relative size of the conformal invariant $\int \sigma_{2}\left(A_{g}\right) d v_{g}$ compared with the Euler number.

Theorem $4.3(28])$. Suppose $(M, g)$ is a closed 4-manifold with $\Lambda(M, g)>0$.

(i) If $\int_{M} \sigma_{2}\left(A_{g}\right) d v_{g}>\frac{1}{4} \int_{M}\left|W_{g}\right|^{2} d v_{g}$, then $M$ is diffeomorphic to $\left(S^{4}, g_{c}\right)$ or $\left(\mathbb{R} P^{4}, g_{c}\right)$.

(ii) If $M$ is not diffeomorphic to $\left(S^{4}, g_{c}\right)$ or $\left(\mathbb{R} P^{4}, g_{c}\right)$ and $\int_{M} \sigma_{2}\left(A_{g}\right) d v_{g}=$ $\frac{1}{4} \int_{M}\left|W_{g}\right|^{2} d v_{g}$, then either

(a) $(M, g)$ is conformally equivalent to $\left(\mathbb{C} P^{2}, g_{F S}\right)$, or

(b) $(M, g)$ is conformally equivalent to $\left(\left(S^{3} \times S^{1}\right) / \Gamma, g_{\text {prod }}\right)$.

It is natural to ask whether this result may be extended to further classify the class of 4-manifolds with metrics belonging to the cone $\Gamma_{k}^{+}$.

There is recent progress by Gursky-Viaclovsky ([63]) on the general solvability of the $\sigma_{k}$ equations when $k>n / 2$ in dimensions three and four. In particular, they introduced an invariant called the k-maximal volume which is an analogue of the Sobolev quotient for conformal metrics belonging to $\Gamma_{k}^{+}$that is always less 
than or equal to that of the standard spheres, and in the case of strict inequality they were able to prove the existence of metric extremizing the k-maximal volume solves the $\sigma_{k}$ equation. In particular they were able to demonstrate in dimension four that the k-maximal volume is strictly smaller than that of the sphere if the conformal structure is different from the standard sphere, and in dimension three such a characterization is still to be verified for $k=2,3$.

In the case when $(M, g)$ is locally conformally flat, there is a lot of progress in understanding the structure of the $\sigma_{k}$ equation when the conformal structure admits metrics whose Schouten tensor belongs to the cone $\Gamma_{k}^{+}$. This is largely due to the result of Schoen-Yau ([95]) which assures that the developing map embeds the holonomy cover as a domain in $S^{n}$ so that the method of moving planes may be used to derive a priori estimates for such equations. In particular a recent series of work of A. Li and Y. Li ([75, [76]) extends the result of $([27$, 28] $)$ to classify all of the solutions of the equation $\sigma_{k}\left(A_{g}\right)=1$ on $\mathbb{R}^{n}$, thus providing a priori estimates for this equation in the locally conformally flat case. In addition, recent work of Guan-Wang ([57]) applied the heat flow associated to the $\sigma_{k}(k \neq n / 2)$ equation to derive the conformally invariant Sobolev inequality for locally conformally flat manifolds, while we have extended the result for the remaining case $k=n / 2$ in general even dimensions.

In general, the geometric implications of the study of $\sigma_{k}$ for manifolds of dimensions greater than four remain open.

\section{Boundary operator, COHn-Vossen InEQUality}

To develop the analysis of the $Q$-curvature equation, it is helpful to consider the associated boundary value problems. In the case of a compact surface with boundary $\left(N^{2}, M^{1}, g\right)$ where the metric $g$ is defined on $N^{2} \cup M^{1}$, the Gauss-Bonnet formula becomes

$$
2 \pi \chi(N)=\int_{N} K d v+\oint_{M} k d \sigma,
$$

where $k$ is the geodesic curvature on $M$. Under conformal change of metric $g_{w}$ on $N$, the geodesic curvature changes according to the equation

$$
\frac{\partial}{\partial n} w+k=k_{w} e^{w} \text { on } \mathrm{M} \text {. }
$$

The boundary value problem for the Yamabe equation was treated by Escobar [42]. On a 4-manifold with boundary $\left(N^{4}, M^{3}, g\right)$, we introduced in ([29]) a third order boundary operator $P_{3}$ along with the boundary curvature invariant $T$. The key property of $P_{3}$ is that it is conformally covariant of bidegree $(0,3)$ when operating on functions defined in a neighborhood of the boundary of compact 4-manifolds, and under conformal change of metric $g_{w}=e^{2 w} g$ on $N^{4}$ we have at the boundary $M^{3}$

$$
\left(P_{3}\right)_{g} w+T_{g}=T_{g_{w}} e^{3 w} .
$$

We refer the reader to ([29]) for the precise definitions of $P_{3}$ and $T$ and will here only mention that on $\left(B^{4}, S^{3}, d x\right)$, where $B^{4}$ is the unit ball in $\mathbb{R}^{4}$, we have

$$
P_{4}=(-\Delta)^{2}, P_{3}=-\left(\frac{1}{2} \frac{\partial}{\partial n} \Delta+\tilde{\Delta} \frac{\partial}{\partial n}+\tilde{\Delta}\right) \quad \text { and } T=2,
$$

where $\tilde{\Delta}$ is the intrinsic boundary Laplacian on $M$. 
In this case the Chern-Gauss-Bonnet formula may be expressed as:

$$
8 \pi^{2} \chi(N)=\int_{N}\left(\frac{1}{4}|W|^{2}+Q_{4}\right) d v+\oint_{M}(\mathcal{L}+T) d \sigma
$$

where $\mathcal{L}$ is a third order boundary curvature invariant that transforms by scaling under conformal change of metric.

The boundary version (5.5) of the Gauss-Bonnet-Chern formula can be used to give an extension of the well known Cohn-Vossen-Huber formula. Let us recall ([39, 70]) that a complete surface $\left(N^{2}, g\right)$ with Gauss curvature in $L^{1}$ has a conformal compactification $\bar{N}=N \cup\left\{q_{1}, \ldots, q_{l}\right\}$ as a compact Riemann surface and

$$
2 \pi \chi(N)=\int_{N} K d A+\sum_{k=1}^{l} \nu_{k},
$$

where at each end $q_{k}$, take a conformal coordinate disk $\left\{|z|<r_{0}\right\}$ with $q_{k}$ at its center; then $\nu_{k}$ represents the following limiting isoperimetric constant:

$$
\nu_{k}=\lim _{r \rightarrow 0} \frac{\operatorname{Length}(\{|z|=r\})^{2}}{2 \operatorname{Area}\left(\left\{r<|z|<r_{0}\right\}\right)} .
$$

This result can be generalized to dimension $n=4$ for locally conformally flat metrics. As we mentioned previously, the developing map of locally conformally flat manifolds having nonnegative Yamabe invariants realizes the holonomy cover as a domain $\Omega$ in the n-sphere $S^{n}$, and in addition, the complement of $\Omega$ has small Hausdorff dimension: $\operatorname{dim}\left(S^{n} \backslash \Omega\right) \leq \frac{n-2}{2}([95])$. It is possible to further constrain the topology as well as the end structure of such manifolds by imposing the natural condition that the $Q$-curvature is in $L^{1}$.

Theorem 5.1 ([30]). Suppose $\left(M^{4}, g\right)$ is a complete locally conformally flat manifold satisfying the conditions:

(i) the scalar curvature $R_{g}$ is bounded between two positive constants and $\left|\nabla_{g} R_{g}\right|$ is also bounded;

(ii) the Ricci curvature is bounded below;

(iii) $\int_{M}\left|Q_{g}\right| d v_{g}<\infty$;

then

(a) if $M$ is simply connected, it is conformally equivalent to $S^{4}-\left\{q_{1}, \ldots, q_{l}\right\}$ and we have

$$
4 \pi^{2} \chi(M)=\int_{M} Q_{g} d v_{g}+4 \pi^{2} l
$$

(b) if $M$ is not simply connected, and we assume in addition that its fundamental group is realized as a geometrically finite Kleinian group without torsion, then we conclude that $M$ has a conformal compactification $\bar{M}=M \cup\left\{q_{1}, \ldots, q_{l}\right\}$ and equation (5.8) holds.

This result gives a geometric interpretation to the $Q$-curvature integral as measuring an isoperimetric constant. A key element is an estimate for conformal metrics $e^{2 w}|d x|^{2}$ defined over domains $\Omega \subset \mathbb{R}^{4}$ satisfying the conditions of Theorem 5.1 that must have a uniform blow-up rate near the boundary:

$$
e^{w(x)} \cong \frac{1}{d(x, \partial \Omega)} .
$$


In the thesis of H. Fang ([44]) there is an analogue of this result in which the condition (iii) is replaced by

$$
\int\left|\sigma_{2}\right| d v<\infty
$$

This result has an appropriate generalization to higher even dimensional situations, in which one has to impose additional curvature bounds to control the lower order terms in the integral. One such extension is also contained in the thesis of H. Fang (44]). For conformal structures which are not necessarily locally conformally flat, there is an extension of Theorem 5.1 by G. Carron and M. Herzlich (19]).

It is possible to constrain the size of the complement for conformal metrics $g \in \Gamma_{k}^{+}$. For example, in the thesis of M. González ([50]), she studies the singular set of conformal metrics on domains in $R^{n}$ belonging to $\Gamma_{k}^{+}$. In ([50]), she extends the argument of (23]) to show the existence of complete conformal metrics on $\Omega \subset S^{n}$ belonging to $\Gamma_{k}^{+}$with suitable bounds on the Ricci tensor implying $\operatorname{dim}\left(S^{n} \backslash \Omega\right)<$ $\frac{n-2 k}{2}$. As a consequence, she obtains the vanishing theorem for certain homotopy groups. This result can be applied to classify certain Kleinian groups in space: a compact conformally flat manifold $\left(M^{n}, g\right)$ with $g \in \Gamma_{k}^{+}$for $2 k>n-2$ is a Schottky group.

The regularity property of the $\sigma_{k}(A)$ is apparently better than that of the $\sigma_{k}\left(\nabla^{2} v\right)$ equation. Much remains to be explored in this direction.

\section{Conformal covariant operator and Q-Curvature}

So far in these lecture notes, we have only discussed the second order differential operators (the Laplace operator on compact surfaces and the conformal Laplace operator on manifolds in dimension $n \geq 3$ ) and some fourth order operators (Paneitz operator on dimension four and the conformal Paneitz operator on dimensions $n \neq 4)$ with the conformal covariant properties as specified in (3.3). We now discuss some general existence results of such operators and their corresponding curvature invariants.

We start with a fundamental paper by C. Fefferman and R. Graham (45]), in which they systematically construct local conformal invariants. They introduce the concept of ambient metric, which is not only an effective tool for computation, but also suggests many interesting questions. Here we will very briefly describe a main step in their construction. First we recall some definitions. Given a Riemannian metric $g$, we denote $[g]$ as the conformal structure consisting of all metrics conformal to $g$, i.e. the collection of all metrics $g_{w}=e^{2 w} g$ for $w \in C^{\infty}(M)$. Suppose $\mathrm{X}$ is a manifold with boundary $(M,[g])$. A Riemannian metric $g^{+}$on $X$ is said to be conformally compact if there is a defining function $x \in C^{\infty}(\bar{X})$ satisfying $x>0$ in $X, x=0$ and $d x \neq 0$ on $M$ with $x^{2} g^{+}$a smooth extension to $\bar{X}$ and when restricted to tangent space TM, $x^{2} g^{+} \in[g]$. In this case, we call $(M,[g])$ the conformal infinity of $X$.

We remark that the above definition is independent of the defining function. Given a conformally compact manifold $\left(X^{n+1}, M^{n}, g^{+}\right)$, we say it is conformally compact Einstein if in addition, $g^{+}$is Einstein.

The conformally compact Einstein manifold is of current interest in the physics literature. The Ads/CFT correspondence proposed by Maldacena ([77]) involves string theory and super-gravity on such $X$ and super-symmetric conformal field 
theory on $M$. Here we will only describe some of the mathematical developments related to conformal geometry.

Given $\left(M^{n}, g\right)$ a compact Riemannian manifold of dimension $n$, denote $M^{+}=$ $M \times[0,1]$, and identify $M$ with $\partial M^{+}=M \times 0$. In ([45]), a metric $g^{+}$defined on $M^{+}$is called a Poincaré metric if

(i) $g^{+}$has $[g]$ as conformal infinity,

(ii) $\operatorname{Ric}\left(g^{+}\right)=-n g^{+}$.

Some further computation shows that a Poincaré metric $g^{+}$in an appropriate coordinate system $\left(\xi_{1}, \xi_{2}, \ldots, \xi_{n}, x\right)$, where $\xi=\left(\xi_{1}, \xi_{2}, \ldots, \xi_{n}\right)$ denotes a point in $M$, can be written as

$$
g^{+}=x^{-2}\left(d x^{2}+\sum_{i, j=1}^{n} g_{i j}^{+}(\xi, x) d \xi_{i} d \xi_{j}\right) .
$$

We need to introduce the additional assumption:

(iii) when written in the form $(6.1), g_{i j}^{+}$is an even function of $x$.

The main result in ([45]) is:

Theorem 6.1. (a) In case $n$ is odd, up to a diffeomorphism fixing $M$, there is a unique formal power series solution of $g^{+}$to (i)-(iii).

(b) In case $n$ is even, if one replaces (ii) by (ii $\left.{ }^{\prime}\right)$ :

(ii ) Along $M$, the components of $\operatorname{Ric}\left(g^{+}\right)+n g^{+}$vanish to order $n-2$ in power series of $x$, then there is a formal power series solution for $g^{+}$.

The construction of the Poincare metric is actually accomplished via the construction of a Ricci flat metric, called the ambient metric on the manifold $\tilde{G}$, where $\tilde{G}=G \times(-1,1)$ and $G$ is the metric bundle

$$
G=\left\{\left(\xi, t^{2} g(\xi)\right): \xi \in M, t>0\right\}
$$

of the bundle of symmetric 2 tensors $S^{2} T^{*} M$ on $M$. The conformal invariants are then contractions of $\left(\tilde{\nabla}^{k_{1}} \tilde{R} \otimes \tilde{\nabla}^{k_{2}} \tilde{R} \otimes \ldots \ldots . \tilde{\nabla}^{k_{l}} \tilde{R}\right)$ restricted to $T M$ where $\tilde{R}$ denotes the curvature tensor of the ambient metric.

A model example is given by the standard sphere $\left(S^{n}, g\right)$. Introduce the projective coordinates $\left(p, p_{0}\right)$ where $p=\left(p_{1}, p_{2}, \ldots, p_{n+1}\right) \in \mathbb{R}^{n+1}$ so that $S^{n}=$ $\left\{\sum_{1}^{n+1} \xi_{k}^{2}=1\right\}$ goes over to

$$
G=\left\{\sum_{1}^{n+1} p_{k}^{2}-p_{0}^{2}=0\right\}
$$

under $\xi_{k}=p_{k} / p_{0}(1 \leq k \leq n+1)$. In the Minkowski space

$$
R^{n+1,1}=\left\{\left(p, p_{0}\right), \mid p \in \mathbb{R}^{n+1}, p_{0} \in \mathbb{R}\right\}
$$

with the Lorentz metric

$$
\tilde{g}=|d p|^{2}-d p_{0}^{2},
$$

the standard hyberbolic space is realized as the quadric $H^{n+1}=\left\{|p|^{2}-p_{0}^{2}=-1\right\} \subset$ $R^{n+1,1}$, and the hyperbolic metric $g_{H}=\tilde{g} \mid H^{n+1}$ is given by

$$
g_{H}=\frac{1}{1+|p|^{2}}(d|p|)^{2}-|p|^{2} g .
$$


An alternative form is to view $\left(H^{n+1}, g_{H}\right)$ as the standard Poincaré ball

$$
\left(H^{n+1},\left(\frac{2}{1-|y|^{2}}\right)^{2}|d y|^{2}\right) .
$$

We can then view $\left(S^{n},[g]\right)$ as the compactification of $H^{n+1}$ using the defining function

$$
x=2 \frac{1+|y|}{1-|y|}
$$

to have $g_{H}$ appear in the form of (6.1):

$$
g_{H}=g^{+}=x^{-2}\left(d x^{2}+\left(1-\frac{x^{2}}{4}\right)^{2} g\right) \text {. }
$$

Based on the construction above, in ([53]), Graham, Jenne, Mason and Sparling have shown the existence of conformal covariant operator $P_{2 k}^{n}$ of order $2 k$ with leading symbol $(-\Delta)^{k}$ defined on an n-dimensional manifold, which is of bi-degree $\left(\frac{n-2 k}{2}, \frac{n+2 k}{2}\right)$, where $\mathrm{k}$ can be any positive integer when $\mathrm{n}$ is odd, but $2 k \leq n$ when $n$ is even. In ([53]; see also [47]), $P_{2 k}^{n}$ is identified with the restriction of $\tilde{P}_{2 k}=\tilde{\Delta}^{k}$ on the ambient space.

We remark that the result above does not assert the uniqueness of the operators $P_{2 k}^{n}$. In fact, if $k$ is a multiple of 2 , then one can add a scale multiple of $|W|^{k}$ to $P_{2 k}^{n}$, where $W$ is the Weyl tensor, without disturbing the conformal covariance of the operator. Yet on $\mathbb{R}^{n}$, the operator is unique and is equal to $(-\Delta)^{k}$. Also, the explicit expression of $P_{2 k}^{n}$ on the standard sphere is known ([11], [8]); hence the explicit formula is also known for manifolds with an Einstein metric. (This latter fact the author learned from T. Branson and R. Graham.)

A related problem is the expression of the $Q$-curvature associated with such operators. Of particular interest is when $n$ is even and $k=\frac{n}{2}$, as we know that when $n=2$ or 4 , such $Q$-curvature is part of the integrand in the Chern-GaussBonnet formula. Assume $n$ is even; then for $2 k \neq n$, one may obtain $Q_{2 k}^{n}$ through the relation $P_{2 k}^{n}(1)=c(n, k) Q_{2 k}^{n}$ for some constant $c(n, k)([53])$, but such relation fails when $k=\frac{n}{2}$. In ([10]), Branson justified the existence of $Q_{n}^{n}$ by a dimension continuation (in $n$ with $k$ fixed) argument from $Q_{2 k}^{n}$; in the recent article by R. Graham and Zworski ([54]) this argument is replaced by the analytic continuation of a spectral parameter. There is also construction of the $Q$-curvature by Gover and Peterson ([51]) using tractor calculus.

We now briefly describe the work in $([54)$.

Supposing $\left(X, g^{+}\right)$is an $n+1$ dimensional manifold with a Poincaré metric and $\left(M^{n},[g]\right)$ a conformal infinity as described above, we say such $g^{+}$is an asymptotically hyperbolic metric. Spectral theory on such spaces has been well studied for example by Mazzeo and Mazzeo-Melrose (78, [81]). A basic fact is that the spectrum $\sigma\left(-\Delta_{g^{+}}\right)$is given by

$$
\sigma\left(-\Delta_{g^{+}}\right)=\left[\left(\frac{n}{2}\right)^{2}, \infty\right) \cup \sigma_{p p}\left(-\Delta_{g^{+}}\right) \text {, with } \sigma_{p p}\left(-\Delta_{g^{+}}\right) \subset\left(0,\left(\frac{n}{2}\right)^{2}\right),
$$

where the pure point spectrum $\sigma_{p p}\left(-\Delta_{g^{+}}\right)$(the set of $L^{2}$ eigenvalues) is finite.

Suppose that $x$ is a defining function associated with a choice of metric $g \in[g]$ on $M$ as before. One considers the asymptotic Dirichlet problem at infinity for the Poisson equation

$$
\left(-\Delta_{g+}-s(n-s)\right) u=0
$$


Based on earlier works on the resolvents, assuming further that when $n$ is even that $g^{+}$satisfies the evenness assumption (iii) as in the statement of Theorem 6.1, Graham and Zworski proved that there is a meromorphic family of solutions $u(s)=$ $\wp(s) f$ such that when Res $>\frac{n}{2}$,

$$
\begin{aligned}
\wp(s) f=F x^{n-s}+G x^{s} & \text { if } s \notin n / 2+N \\
\wp(s) f=F x^{n / 2-k}+H x^{n / 2+k} \log x & \text { if } s=n / 2+k, k \in N,
\end{aligned}
$$

where $F, G, H \in C^{\infty}(X),\left.F\right|_{M}=f$, and $F, G \bmod O\left(x^{n}\right)$ are even in $x$. Moreover, if $n / 2-s$ is an integer, then $\left.H\right|_{M}$ is locally determined by $f$ and $g$. However, if $n / 2-s$ is not an integer, then $\left.G\right|_{M}$ is globally determined by $f$ and $g$. The scattering operator is defined as:

$$
S(s) f=\left.G\right|_{M} .
$$

One of the main results in [54] is

Theorem 6.2. Let $\left(X^{n+1}, M^{n}, g^{+}\right)$be an asymptotically hyperbolic metric with $\left(M^{n},[g]\right)$ as conformal infinity. Suppose $k \in \mathbb{N}$ and $k \leq \frac{n}{2}$ if $n$ is even and $s(n-s)$ not in $\sigma_{p p}\left(-\Delta_{g^{+}}\right)$. Then the scattering matrix $S(s)$ has a simple pole at $s=\frac{n}{2}+k$ and

$$
c_{k} P_{2 k}^{n}=-\operatorname{Res}_{s=\frac{n}{2}+k} S(s), \text { where } c_{k}=(-1)^{k}\left[2^{2 k} k !(k-1) !\right]^{-1} .
$$

When $2 k \neq n, P_{2 k}^{n}(1)=c(n, k) Q_{2 k}^{n}$.

When $2 k=n, c_{\frac{n}{2}} Q_{n}=S(n) 1$.

We remark that the curvatures $Q_{n}^{n}$ thus defined are unique. For $n=2, Q_{2}^{2}=\frac{R}{2}$; for $n=4, Q_{4}^{4}=2 Q$ for the $Q$-curvature defined in section 3. Denoting $Q_{n}^{n}=Q_{n}$, the general formula for $Q_{n}$ can be computed recursively, but the computation is very complicated and has so far been carried out for $n=6$ and $n=8$ ([51]; also private communication from R. Graham). Here we summarize some known facts about $Q_{n}$ for $n$ even:

(a) $Q_{n}$ is a conformal density of weight -n; i.e. with respect to the dilation $\delta_{t}$ of metric $g$ given by $\delta_{t}(g)=t^{2} g$, we have $\left(Q_{n}\right)_{\delta_{t} g}=t^{-n}\left(Q_{n}\right)_{g}$.

(b) $\int_{M^{n}}\left(Q_{n}\right)_{g} d v_{g}$ is conformally invariant.

(c) Under the conformal change of metric $g_{w}=e^{2 w} g$, we have

$$
\left(P_{n}\right)_{g} w+\left(Q_{n}\right)_{g}=\left(Q_{n}\right)_{g_{w}} e^{n w} .
$$

(d) When $\left(M^{n}, g\right)$ is locally conformally flat, then $\left(Q_{n}\right)_{g}=a_{n} \sigma_{\frac{n}{2}}\left(A_{g}\right)$, where $A_{g}, \sigma_{k}$ are defined in section 4 .

(e) More significantly, Alexakis has announced a proof of the following conjectural expression of $Q$ :

$$
Q_{n}=a_{n} \text { Pfaffian }+J+\operatorname{div}\left(T_{n}\right),
$$

where Pfaffian is the Euler class density, which is the integrand in the Chern-GaussBonnet formula; $J$ is a pointwise conformal invariant; and $\operatorname{div}\left(T_{n}\right)$ is a divergence term.

(f) In his thesis, Alexakis ([2]; see also [47], for special case [51]) has extended the existence of conformal covariant operators to conformal densities of weight $\gamma$, where 
$\gamma \neq\left(-\frac{n}{2}\right)+k$, and other than $l$, where $k$ is a positive integer and $l$ a nonnegative integer. An example of such an operator is:

$$
2 P(f)=\nabla^{i}\left(\|W\|^{2} \nabla^{i} f\right)+\frac{n-6}{n-2}\|W\|^{2} \Delta f,
$$

with the corresponding Q-curvature explicitly written.

In ([47]), Fefferman and Hirachi have also extended the construction of conformal covariant operators and $Q$-curvature to $C R$ manifolds. We refer readers to the articles (46], 47]) and also the lecture notes of Branson, Eastwood and Gover at the AIM conference this past summer for the latest developments in this subject.

\section{Renormalized volume}

The volume of any conformally compact manifold is infinite. An appropriate renormalization of volume for conformally compact Einstein manifolds gives rise to new volume invariants. In the physics setting, it arises from a procedure outlined by Witten ([101]) and by Gubser, Klebanov and Polyakov ([58]). The volume renormalization was carried out by Henningson and Skenderis (68]). The reader is also referred to the article by Graham ([52]) for a mathematical approach to the subject based on the Poincaré metric construction discussed in section 6 . In this section, we will discuss the connection between the renormalized volume and $Q$-curvature.

Recall a conformally compact Einstein manifold is a manifold $\left(X^{n+1}, M^{n}, g^{+}\right)$, such that $g^{+}$is an Einstein manifold and $\left(M^{n},[g]\right)$ is the conformal infinity of $X$. We have normalized so that $\operatorname{Ric}\left[g^{+}\right]=-n g^{+}$on $X$. Suppose we choose a defining function $x$ so that in a neighborhood of $M$ we have

$$
g^{+}=x^{-2}\left(d x^{2}+g_{x}\right),
$$

where $g_{x}$ is a parameter family of metrics on $M$ with $g=\left.x^{2} g^{+}\right|_{x=0}, g \in[g]$ is a representative of metrics associated with the defining function $x$. The renormalized volume $V$ is defined as the finite part in the expansion of $\operatorname{Vol}_{g^{+}}(x>\epsilon)$ as $\epsilon \rightarrow 0$.

$$
\operatorname{Vol}_{g^{+}}(\{x>\epsilon\})=c_{0} \epsilon^{-n}+c_{2} \epsilon^{-n+2}+\cdots+c_{n-1} \epsilon^{-1}+V+o(1)
$$

for $n$ odd, and

$$
\operatorname{Vol}_{g^{+}}(\{x>\epsilon\})=c_{0} \epsilon^{-n}+c_{2} \epsilon^{-n+2}+\cdots+c_{n-2} \epsilon^{-2}+L \log \frac{1}{\epsilon}+V+o(1)
$$

for $n$ even.

The renormalized volume $V$ is independent of the conformal representative $g$ on the boundary when $n$ is odd, and $L$ is independent of the conformal representative when $\mathrm{n}$ is even. The dependence of $V$ on the choice of $\mathrm{g}$ for $\mathrm{n}$ even is called the holographic anomaly (cf. [68], [52]). Using the connection with the scattering matrix, Graham and Zworski have also identified $L$ in terms of the $Q$-curvature.

Theorem 7.1 (54]). When $n$ is even,

$$
L=2 c_{\frac{n}{2}} \int_{M} Q_{n} d v_{g}
$$

where $c_{\frac{n}{2}}$ is the constant defined in (6.4).

In subsequent work, Fefferman and Graham gave a different proof of the above result. Furthermore they have extended the notion of $Q_{n}$ to manifolds with odd dimension $\mathrm{n}$ in the following sense: 
Theorem 7.2 (46]). There is a unique smooth function $v$ defined on $X$ solving

$$
-\Delta_{g^{+}}(v)=n
$$

and with the asymptotic

$$
v= \begin{cases}\log x+A+B x^{n} \log x & \text { for } n \text { even } \\ \log x+A+B x^{n} & \text { for } n \text { odd }\end{cases}
$$

where $A, B \in C^{\infty}(X)$ are even $\bmod O\left(x^{\infty}\right)$ and $\left.A\right|_{M}=0$. Moreover

(i) if $n$ is even, then

$$
\left.B\right|_{M}=-2 c_{\frac{n}{2}} Q_{n}
$$

hence

$$
L=2 c_{\frac{n}{2}} \int_{M} Q_{n}
$$

(ii) If $n$ is odd, then

$$
\left.B\right|_{M}=-\left.\frac{d}{d s}\right|_{s=n} S(s) 1
$$

and if one defines $Q_{n}\left(g^{+},[g]\right)$ to be

$$
Q_{n}\left(g^{+},[g]\right)=\left.k_{n} B\right|_{M}, \text { where } k_{n}=2^{n} \frac{\Gamma(n / 2)}{\Gamma(-n / 2)},
$$

then

$$
k_{n} V=\int_{M} Q_{n}\left(g^{+},[g]\right) d v_{g},
$$

where $V$ is the renormalized volume.

We remark that when $\mathrm{n}$ is odd, the $Q$-curvature thus defined depends not only on the boundary metric $g$ on $M$ but also on the extension of $g^{+}$on $X$.

We now restrict to $n=3$ and illustrate the connection between the $Q$-curvature and the boundary operator $P_{b}$ and curvature $T$ which have been discussed in section 5 . Recall in section 5 that we mentioned that in ([29]), on an arbitrary compact Riemannian 4-manifold $\left(X^{4}, M^{3}, g^{+}\right)$with boundary, a third order boundary operator $P_{b}$ and a third order boundary curvature $T$ were introduced which satisfy:

$$
\begin{aligned}
& \left(P_{b}\right)_{g_{w}}=e^{-3 w}\left(P_{b}\right)_{g}, \quad \text { on } \mathrm{M} \text { and } \\
& \left(P_{b}\right)_{g} w+T_{g}=T_{g_{w}} e^{3 w} \quad \text { on M. }
\end{aligned}
$$

We have also re-organized the terms in the integrand of the Chern-Gauss-Bonnet formula for 4-manifolds with boundary into the following form:

$$
8 \pi^{2} \chi(X)=\int_{X^{4}}\left(\frac{1}{4}|W|^{2}+Q_{4}\right) d v+2 \int_{M^{3}}(\mathcal{L}+T) d \sigma,
$$

where $\mathcal{L}$ is a pointwise conformal invariant term on the boundary of the manifold.

While for a general $\left(X^{4}, M^{3}, g^{+}\right)$, the formula for $\left(P_{b}\right)_{g}, T_{g}$ and $\mathcal{L}$ are quite complicated, the expressions become very simple when the boundary is umbilical and is totally geodesic:

$$
\begin{gathered}
\left(P_{b}\right)_{g}=-\left.\frac{1}{2} \frac{\partial}{\partial n} \Delta_{g^{+}}\right|_{M}+\tilde{\Delta} \frac{\partial}{\partial n}-\left(F-\frac{1}{3} R\right) \frac{\partial}{\partial n}, \\
T_{g}=\left.\frac{1}{12} \frac{\partial R}{\partial n}\right|_{M},
\end{gathered}
$$

where $F=R-\alpha N \alpha N, N=\frac{\partial}{\partial n}$, and in this case $\mathcal{L}$ vanishes. 
In the special case when $\left(X^{4}, M^{3}, g^{+}\right)$is a conformally compact Einstein manifold, we now consider the metric $e^{2 v} g^{+}$, where $v$ is the function satisfying the equation (7.5). We make the following observation:

Theorem 7.3 ([32]). (i) $\left(Q_{4}\right)_{e^{2 v} g^{+}}=0$, and

(ii) $Q_{3}\left(e^{2 v} g^{+},\left[e^{2 v} g\right]\right)=\left.3 B\right|_{x=0}=T_{e^{2 v}}$; as a consequence we have

$$
\int_{X^{4}} \sigma_{2}\left(A_{e^{2 v} g^{+}}\right)=\int_{X^{4}}\left(Q_{4}\right)_{e^{2 v} g^{+}}+2 \int_{M^{3}} T_{e^{2 v} g}=6 \mathrm{~V} .
$$

Combining the results in Theorems 7.2 and 7.3, we have given a different proof of the following result of Anderson (3]) relating the renormalized volume to the Pffafian integral.

Theorem 7.4. Suppose that $\left(X^{4}, g^{+}\right)$is a conformally compact Einstein manifold. Let us set $\bar{g}=e^{2 v} g^{+}$; then

$$
8 \pi^{2} \chi\left(X^{4}\right)=\frac{1}{4} \int_{X^{4}}|W|^{2} d v_{\bar{g}}+\int_{X^{4}} \sigma_{2}\left(A_{\bar{g}}\right)=\frac{1}{4} \int_{X^{4}}|W|^{2} d v_{g^{+}}+6 V .
$$

Combining the conjecture (e) of the structure of general $Q$-curvature in section 6 and the observation made above, one can extend the formula for the renormalized volume to any $\left(X^{n+1}, M^{n}, g^{+}\right)$conformally compact Einstein manifold for $n$ odd. In particular one obtains as a special case a formula of Epstein (appendix A in [87]) for the renormalized volume of any hyperbolic manifold as a multiple of the Euler number of the manifold. It turns out ([32]) that when $n$ is even, one can instead relate the renormalized volume to the conformal primitive of the $Q$-curvature.

This understanding of the relation between the renormalized volume and the integral in the Chern-Gauss-Bonnet formula also allows us to translate some of the results in ([59], 26], 28]) from compact 4-manifolds without boundary to the setting of conformal compact Einstein 4-manifolds.

Theorem 7.5 ([31]). Suppose $\left(X^{4}, M^{3}, g^{+}\right)$is a conformal compact Einstein manifold, and assume further that the conformal infinity $\left(M^{3},[g]\right)$ has positive Yamabe invariant. Then

(i)

$$
V \leq \frac{4}{3} \pi^{2}
$$

with equality holding if and only if $\left(X^{4}, g^{+}\right)$is the hyperbolic space $\left(H^{4}, g_{H}\right)$, and therefore $\left(M^{3}, g\right)$ is the standard 3-sphere.

(ii) If

$$
V>\frac{1}{3} \frac{4 \pi^{2}}{3} \chi(X)
$$

then $X$ is homeomorphic to the 4 -ball $B^{4}$ up to a finite cover.

(iii) If

$$
V>\frac{1}{2} \frac{4 \pi^{2}}{3} \chi(X)
$$

then $X$ is diffeomorphic to $B^{4}$ and $M$ is diffeomorphic to $S^{3}$.

A crucial step in the proof of the theorem above is an earlier result by J. Qing (91]), which builds upon some earlier estimates of J. Lee [3] on the subject. 
Theorem 7.6 (91]). Suppose $\left(X^{n+1}, M^{n}, g^{+}\right)$is a conformal compact Einstein manifold, and with the Yamabe constant of $\left(M^{n},[g]\right)$ being positive, there is a positive eigenfunction u satisfying

$$
-\Delta_{g^{+}} u=(n+1) u \text { on } X^{n+1},
$$

so that $\left(X^{n+1}, u^{-2} g^{+}\right)$is a compact manifold with totally geodesic boundary and the scalar curvature is greater than or equal to $\frac{n+1}{n-1} R_{g}$, where we have taken $g \in[g]$ to be the Yamabe metric.

In ([91]) the above theorem was used to establish the rigidity result that any conformal compact Einstein manifold with conformal infinity the standard n-sphere is the hyperbolic $n+1$ space. Given $\left(M^{n},[g]\right)$ in general, both the existence and the uniqueness problems of a conformal compact Einstein manifold with $\left(M^{n},[g]\right)$ as conformal infinity remain open.

\section{REFERENCES}

[1] D. Adams; A sharp inequality of J. Moser for higher order derivatives, Ann. of Math. (2), 128 (1988), 385-398. MR0960950 (89i:46034)

[2] S. Alexakis; On conformally invariant differential operators in odd dimensions, Proc. Nat. Acad. Sci, no. 8, 100 (2003), 4409-4410. MR1971495 (2004g:58046)

[3] M. Anderson; $L^{2}$ curvature and volume renormalization of the AHE metrics on 4-manifolds, Math. Res. Lett., 8 (2001), 171-188. MR,1825268 (2002k:53079)

[4] T. Aubin; Problemes isoperimetriques et espaces de Sobolov, J. Diff. Geom., 11 (1976), 573-598. MR0448404 (56:6711)

[5] T. Aubin; Equations differentielles non lineaires et probleme de Yamabe concernant la courbure scalaire, J. Math. Pures Appl., 55 (1976), 269-296. MR.0431287 (55:4288)

[6] T. Aubin; Meilleures constantes dans le theorem d'inclusion de Sobolev et un theorem de Fredholme non lineaire pour la transformation conforme de la courbure scalaire, J. Funct. Anal., 32 (1979), 148-174. MR0534672 (80i:58043)

[7] A. Bahri and J. M. Coron; The scalar curvature problem on the standard three dimensional sphere, J. Funct. Anal., 95 (1991), 106-172. MR.1087949 (92k:58055)

[8] W. Beckner; Sharp Sobolev inequalities on the sphere and the Moser-Trudinger inequality, Ann. of Math., 138 (1993), 213-242. MR1230930 (94m:58232)

[9] G. Bliss; An integral inequality, J. London Math. Soc., 5 (1930), 44-46.

[10] T. Branson; Differential operators canonically associated to a conformal structure, Math. Scand., 57 (1985), 293-345. MR0832360 (88a:58212)

[11] T. Branson; The functional determinant, Lecture notes series, No. 4, Seoul National University, 1993. MR:1325463 (96g:58203)

[12] T. Branson; Sharp inequalities, the functional determinant and the complementary series, Trans. Amer. Math. Soc., 347 (1995), 3671-3742. MR1316845 (96e:58162)

[13] T. Branson, S.-Y. A. Chang and P. Yang; Estimates and extremals for zeta-function determinants on four-manifolds, Comm. Math. Phys., 149 (1992), no. 2, 241-262. MR 1186028 (93m:58116)

[14] T. Branson and B. Ørsted; Explicit functional determinants in four dimensions, Proc. Amer. Math. Soc., 113 (1991), 669-682. MR1050018 (92b:58238)

[15] S. Brendle; Global existence and convergence for a higher order flow in conformal geometry, Ann. of Math. (2), 158 (2003), no. 1, 323-343. MR1999924 (2004e:53098)

[16] L. A. Caffarelli; A localization property of viscosity solutions to the Monge-Ampère equation and their strict convexity, Ann. of Math. (2), 131 (1990), no. 1, 129-134. MR1038359 (91f:35058)

[17] L. Caffarelli, L. Nirenberg and J. Spruck; The Dirichlet problem for nonlinear second order elliptic equations, III: Functions of the eigenvalues of the Hessian, Acta Math., 155 (1985), no. 3-4, 261-301. MR.0806416 (87f:35098)

[18] L. Caffarelli, B. Gidas and J. Spruck; Asymptotic symmetry and local behavior of semilinear equations with critical Sobolev growth, Comm. Pure Appl. Math., 42 (1989), 271-297. MR $0982351(90 \mathrm{c}: 35075)$ 
[19] G. Carron and M. Herzlich; The Huber theorem for non-compact conformally flat manifolds, Comment. Math. Helv., 77 (2002), 192-220. MF1898398 (2003h:53048)

[20] L. Carleson and S.-Y. A. Chang; On the existence of an extremal function for an inequality of J. Moser, Bull. Sci. Math. (2), 110 (1986), 113-127. MR0878016 (88f:46070)

[21] K.-C. Chang and J. Q. Liu; On Nirenberg's problem, Inter. J. Math., 4 (1993), 35-58. MR.1209959 (94a:58038)

[22] S.-Y. A. Chang, C.-C. Chen and C.-S. Lin; Extremal functions for a mean field equation in two dimension, Lectures on Partial Differential Equations in Honor of Louis Nirenberg's 75th Birthday, Chapter 4, International Press, 2003. MR2055839

[23] S.-Y. A. Chang, F. Hang and P. Yang; On a class of locally conformally flat manifolds, Int. Math. Res. Not. 2004, no. 4, 185-209. MR2040327

[24] S.-Y. A. Chang, M. Gursky and P. Yang; The scalar curvature equation on 2- and 3-spheres, Calculus of Variation, 1 (1993), 205-229. MR.1261723 (94k:53055)

[25] S.-Y. A. Chang, M. Gursky and P. Yang; An equation of Monge-Ampère type in conformal geometry, and four-manifolds of positive Ricci curvature, Ann. of Math., 155 (2002), no. 3, 711-789. MR1923964 (2003j:53048)

[26] S.-Y. A. Chang, M. Gursky and P. Yang; An a prior estimate for a fully nonlinear equation on four-manifolds, J. D'Analyse Math.; Thomas Wolff memorial issue, 87 (2002), 151-186. MR.1945280 (2003k:53036)

[27] S.-Y. A. Chang, M. Gursky and P. Yang; Entire solutions of a fully nonlinear equation, Lectures on Partial Differential Equations in Honor of Louis Nirenberg's 75th Birthday, Chapter 3, International Press, 2003. MR2055838 (2005b:53053)

[28] S.-Y. A. Chang, M. Gursky and P. Yang; A conformally invariant sphere theorem in four dimensions, Publ. Math. Inst. Hautes Études Sci. No. 98, (2003), 105-143. MR2031200 (2005b:53054)

[29] S.-Y. A. Chang and J. Qing; The zeta functional determinants on manifolds with boundary. I-the formula, J. Funct. Anal., 147 (1997), no. 2, 327-362. MR.1454485 (98f:58198)

[30] S.-Y. A. Chang, J. Qing and P. Yang; Compactification for a class of conformally flat 4-manifolds, Invent. Math., 142 (2000), 65-93. MR1784799 (2001m:53061)

[31] S.-Y. A. Chang, J. Qing and P. Yang; On the topology of conformally compact Einstein 4-manifolds, Noncompact problems at the intersection of geometry, analysis, and topology, 49-61, Contemp. Math., 350, Amer. Math. Soc., Providence, RI, 2004. MR2082390

[32] S.-Y. A. Chang, J. Qing and P. Yang; On renormalized volume on conformally compact Einstein manifold, preprint, 2003.

[33] S.-Y. A. Chang and P. Yang; Prescribing Gaussian curvature on $S^{2}$, Acta Math., 159 (1987), 215-259. MR0908146 (88m:35056)

[34] S.-Y. A. Chang and P. Yang; Conformal deformation of metrics on $S^{2}$, J. Diff. Geom., 27 (1988), no. 2, 259-296. MR0925123 (89b:53079)

[35] S.-Y. A. Chang and P. Yang; Extremal metrics of zeta functional determinants on 4manifolds, Ann. of Math., 142 (1995), 171-212. MR.1338677 (96e:58034)

[36] W.X. Chen and C. Li; Classification of solutions of some non-linear elliptic equations, Duke Math. J., no. 3, 63 (1991), 615-622. MR.1121147 (93e:35009)

[37] C.-C. Chen and C.-S. Lin; Topological degree for a mean field equation on Riemann surfaces, Comm. Pure Appl. Math., 56 (2003), no. 12, 1667-1727. MR2001443 (2004h:35065)

[38] C.-C. Chen and C.-S. Lin; Prescribing scalar curvature on $S^{n}$, Part I, A priori estimates, J. Diff. Geom., 57 (2002), 67-171. MR1871492 (2002j:53038)

[39] S. Cohn-Vossen; Kürzest Wege und Totalkrümmung auf Flächen, Compositio Math., 2 (1935), 69-133.

[40] Z. Djadli, E. Hebey and M. Ledoux; Paneitz-type operators and applications, Duke Math. J., 104 (2000), no. 1, 129-169. MR:1769728(2002f:58061)

[41] Z. Djadli, A. Malchiodi and M. O. Ahmedou; Prescribing a fourth order conformal invariant on the standard sphere, Part II: Blowup analysis and applications, Ann. Sc. Norm. Super. Pisa Cl. Sci. (5), 1 (2002), no. 2, 387-434. MR1991145 (2004i:53043)

[42] J. Escobar; The Yamabe problem on manifolds with boundary, J. Diff. Geom., 35 (1992), 21-84. MR 1152225 (93b:53030)

[43] C. Evans; Classical solutions of fully non-linear, convex, second order elliptic equations, Comm. Pure Appl. Math., XXXV (1982), 333-363. MR0649348 (83g:35038) 
[44] H. Fang; A conformal Gauss-Bonnet-Chern inequality for LCF manifolds and related topics, to appear in Calculus of Variations and PDE.

[45] C. Fefferman and C. R. Graham; Conformal invariants, In: Élie Cartan et les Mathématiques d'aujourd'hui. Asterisque (1985), 95-116. MR0837196 (87g:53060)

[46] C. Fefferman and C. R. Graham; Q-curvature and Poincaré metrics, Math. Res. Lett., 9 (2002), nos. 2 and 3, 139-152. MR1909634 (2003f:53053)

[47] C. Fefferman and K. Hiriachi; Ambient metric construction of Q-curvature in conformal and CR geometries, Math. Res. Lett., 10 (2003), 819-831. MR2025058

[48] M. Flücher; Extremal functions for the Trudinger-Moser inequality in 2 dimensions, Comm. Math. Helv., 67 (1992), 471-497. MR1171306 (93k:58073)

[49] L. Gårding; An inequality for hyperbolic polynomials, J. Math. Mech., 8 (1959), 957-965. MR 0113978 (22:4809)

[50] M. Del Mar González; Singular sets of a class of fully non-linear equations in conformal geometry, Ph.D. Thesis, Princeton University, 2004.

[51] A. R. Gover and L. Peterson; Conformally invariant powers of the Laplacian, Q-curvature, and tractor calculus, Comm. Math. Phys., 235 (2003), no. 2, 339-378. MR1969732 (2004d:58047)

[52] C. R. Graham; Volume and area renormalizations for conformally compact Einstein metrics, The Proceedings of the 19th Winter School "Geometry and Physics" (Srní, 1999). Rend. Circ. Mat. Palermo (2) Suppl. No. 63 (2000), 31-42. MR.1758076 (2002c:53073)

[53] C. R. Graham, R. Jenne, L. Mason, and G. Sparling; Conformally invariant powers of the Laplacian, I: existence, J. London Math. Soc. (2), 46 (1992), no. 2, 557-565. MR1190438 (94c:58226)

[54] C. R. Graham and M. Zworski; Scattering matrix in conformal geometry, Invent. Math., 152 (2003), no. 1, 89-118. MR.1965361 (2004c:58064)

[55] B. Guan and J. Spruck; Boundary value problems on $S^{n}$ for surfaces of constant Gauss curvature, Ann. of Math., 138 (1993), 601-624. MR1247995 (94i:53039)

[56] P. Guan, J. Viaclovsky and G. Wang; Some properties of the Schouten tensor and applications to conformal geometry, Trans. Amer. Math. Soc., 355 (2003), no. 3, 925-933 (electronic). MR 1938739 (2003h:53054)

[57] P. Guan and G. Wang; A fully nonlinear conformal flow on locally conformally flat manifolds, J. Reine Angew. Math., 557 (2003), 219-238. MR.1978409 (2004e:53101)

[58] S. S. Gubser, I. R. Klebanov and A. M. Polyakov; Gauge theory correlators from non-critical string theory, Phys. Lett. B, 428 (1998), 105-114. MF,1630766 (99e:81204b)

[59] M. Gursky; The Weyl functional, deRham cohomology and Kahler-Einstein metrics, Ann. of Math. (2), 148 (1998), 315-337. MR1652920 (99i:58036)

[60] M. Gursky; The principal eigenvalue of a conformally invariant differential operator, with an application to semi-linear elliptic PDE, Comm. Math. Phys., 207 (1999), 131-143. MF, 1724863 (2000k:58029)

[61] M. Gursky and J. Viaclovsky; A new variational characterization of three-dimensional space forms, Invent. Math., 145 (2001), 251-278. MR.1872547(2002j:53039)

[62] M. Gursky and J. Viaclovsky; A fully nonlinear equation on four-manifolds with positive scalar curvature, J. Diff. Geom., 63 (2003), 131-154. MR2015262 (2004h:53052)

[63] M. Gursky and J. Viaclovsky; Volume comparison and the $\sigma_{k}-$ Yamabe problem, Adv. Math., 187 (2004), no. 2, 447-487. MF 2078344

[64] R. Hamilton; Four manifolds with positive curvature operator, J. Diff. Geom., 24 (1986), 153-179. MR0862046 (87m:53055)

[65] Z.-C. Han; Prescribing Gaussian curvature on $S^{2}$, Duke Math. J., 61 (1990), 679-703. MR 1084455 (91m:58028)

[66] F. Hang and P. Yang; The Sobolev inequality for Paneitz operator on three manifolds, Calc. Var. Partial Differential Equations, 21 (2004), no. 1, 57-83. MR2078747

[67] E. Hebey and F. Robert; Coercivity and Struwe's compactness for Paneitz type operators with constant coefficients, Calc. of Var., 13 (2001), 491-517. MR1867939 (2003m:58051)

[68] M. Henningson and K. Skenderis; The holographic Weyl anomaly, J. High. Energy Phys., 07 (1998), 023 hep-th/9806087; Holography and the Weyl anomaly, hep-th/9812032. MR.1644988 (99f:81162)

[69] C. W. Hong; A best constant and the Gaussian curvature, Proc. Amer. Math. Soc., 97 (1986), 737-747. MR0845999 (87k:58284) 
[70] A. Huber; On subharmonic functions and differential geometry in the large, Comm. Math. Helv., 32 (1957), 13-72. MR0094452 (20:970)

[71] J. Kazdan and F. Warner; Existence and conformal deformation of metrics with prescribed Gaussian and scalar curvature, Ann. of Math., 101 (1975), 317-331. MR0375153 (51:11349)

[72] N.V. Krylov; Boundedly inhomogeneous elliptic and parabolic equations, Izv. Akad. Nauk. SSSR Ser. Mat., 46 (1982), 487-523; English transl. in Math. USSR Izv., 20 (1983), 459-492. MR 0661144 (84a:35091)

[73] J. Lee; The spectrum of an asymptotically hyperbolic Einstein manifold, Comm. Anal. Geom., 3 (1995), no. 1-2, 253-271. MR.1362652(96h:58176)

[74] Y. Li; Prescribing scalar curvature on $S^{n}$ and related problems, Part II: Existence and compactness, Comm. Pure and Appl. Math., XLIX (1996), 541-587. MF1383201|(98f:53036)

[75] A. Li and Y. Li; A fully nonlinear version of the Yamabe problem and a Harnack type inequality, C. R. Math. Acad. Sci. Paris, 336 (2003), no. 4, 319-324. MR1976311(2004b:53055)

[76] A. Li and Y. Li; On some conformally invariant fully nonlinear equations, Comm. Pure Appl. Math., 56 (2003), no. 10, 1416-1464. MR1988895(2004e:35072)

[77] J. Maldacena; The large $N$-limit of super conformal field theories and supergravity, Adv. Theor. Math. Phys., 2 (1998), 231-252. MF1633016 (99e:81204a)

[78] R. Mazzeo; The Hodge cohomology of a conformally compact metric, JDG, 28 (1988), 309339. MR0961517 (89i:58005)

[79] J. Moser; A sharp form of an inequality by N. Trudinger, Indiana Univ. Math. J., 20 (1971), 1077-1091. MR0301504 (46:662)

[80] J. Moser; On a non-linear problem in differential geometry, Dynamical Systems (Proc. Sympos. Univ. Bahia, Salvador, 1971), 273-280, Academic Press, New York, 1973. MR0339258 (49:4018)

[81] R. Mazzeo and R. Melrose; Meromorphic extension of the resolvent on complete spaces with asymptotically constant negative curvature, J. Funct. Anal., 75 (1987), 260-310. MR0916753 (89c:58133)

[82] E. Onofri; On the positivity of the effective action in a theory of random surfaces, Comm. Math. Phys., 86 (1982), 321-326. MF0677001 (84j:58043)

[83] B. Osgood, R. Phillips, and P. Sarnak; Extremals of determinants of Laplacians, J. Funct. Anal., 80 (1988), 148-211. MR0960228 (90d:58159)

[84] B. Osgood, R. Phillips, and P. Sarnak; Compact isospectral sets of surfaces, J. Funct. Anal., 80 (1988), 212-234. MR0960229 (90d:58160)

[85] K. Okikiolu; The Campbell-Hausdorff theorem for elliptic operators and a related trace formula, Duke Math. J., 79 (1995) 687-722. MR1355181 (96j:58175)

[86] K. Okikiolu; Critical metrics for the determinant of the Laplacian in odd dimensions, Ann. of Math. (2), 153 (2001), no. 2, 471-531. MR.1829756 (2002j:58056)

[87] S. Patterson and P. Perry; The divisor of Selberg's zeta function for Kleinian groups, Duke Math. J., 106 (2001), 321-390. Appendix A by C. Epstein, An asymptotic volume formula for convex cocompact hyperbolic manifolds. MR1813434(2002a:11103)

[88] A. V. Pogorelev; The Dirichlet problem for the multidimensional analogue of the MongeAmpère equation, Dokl. Acad. Nauk. SSSR, 201 (1971), 790-793. English translation, Soviet Math. Dokl., 12 (1971), 1227-1231. MR0293228 (45:2305)

[89] S. Paneitz; A quartic conformally covariant differential operator for arbitrary pseudoRiemannian manifolds, Preprint, 1983.

[90] A. Polyakov; Quantum geometry of bosonic strings, Phys. Lett. B, 103 (1981), 207-210. MR.0623209 (84h:81093a)

[91] J. Qing; On the rigidity for conformally compact Einstein manifolds, Inter. Math. Res. Notices, 21 (2003) 1141-1153, ArXiv: math.DG/0305084. MR1962123 (2004a:53049)

[92] D. B. Ray and I. M. Singer; R-torsion and the Laplacian on Riemannian manifolds, Advances in Math., 7 (1971), 145-210. MR.0295381 (45:4447)

[93] R. Schoen; Conformal deformation of a Riemannian metric to constant scalar curvature, J. Diff. Geom., 20 (1984), 479-495. MR0788292 (86i:58137)

[94] R. Schoen and D. Zhang; Prescribed scalar curvature on the $n$-sphere, Calc. of Vari., 4 (1996), 1-25. MR1379191 (97j:58027)

[95] R. Schoen and S.-T. Yau; Conformally flat manifolds, Kleinian groups and scalar curvature, Invent. Math., 92 (1988), 47-71. MR0931204 (89c:58139) 
[96] T.-L. Soong; Extremal functions for the Moser inequality on $S^{2}$ and $S^{4}$, Ph.D. Thesis, UCLA, 1991.

[97] G. Talenti; Best constant in Sobolev inequality, Ann. Mat. Pura Appl. (4), 110 (1976), 353-372. MR0463908 (57:3846)

[98] N. Trudinger; On imbeddings into Orlicz spaces and some applications, J. Math. Mech., 17 (1967), 473-483. MR 0216286(35:7121)

[99] N. Trudinger; Remarks concerning the conformal deformation of Riemannian structures on compact manifolds, Ann. Scuolo Norm. Sup. Pisa (3), 22 (1968), 265-274. MR0240748 (39:2093)

[100] J. Viaclovsky; Conformal geometry, contact geometry and the calculus of variations, Duke Math. J., 101 (2000), no. 2, 283-316. MR.1738176 (2001b:53038)

[101] E. Witten; Anti-de Sitter space and holography, Adv. Theor. Math. Phys., 2 (1998), 253-290. MR $1633012(99 \mathrm{e}: 81204 \mathrm{c})$

[102] X.-W. Xu and P. Yang; On a fourth order equation in 3-D, ESAIM Control Optim. Calc. Var., 8 (2002), 1029-1042 (electronic). MF,1932985(2003i:53051)

[103] H. Yamabe; On a deformation of Riemannian structures on compact manifolds, Osaka Math. J., 12 (1960), 21-37. MR0125546 (23:A2847)

Department of Mathematics, Princeton University, Princeton, New Jersey 08544

E-mail address: chang@math.princeton.edu 